\title{
Total Synthesis of (-)- and ent-(+)-Vindoline
}

Younggi Choi, Hayato Ishikawa, Juraj Velcicky, Gregory I. Elliott, Michael M. Miller and Dale L. Boger*

Department of Chemistry and The Skaggs Institute for Chemical Biology,

The Scripps Research Institute,

10550 North Torrey Pines Road, La Jolla, California 92037

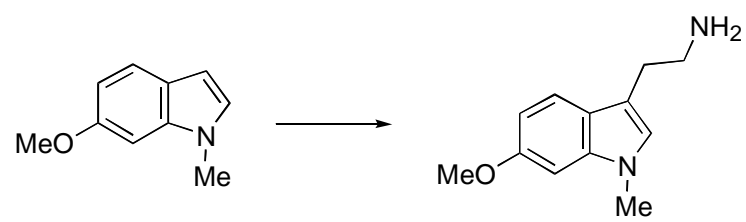

N-Methyl-6-methoxytryptamine (6). This material was prepared as previously detailed ${ }^{S 1}$ from 6-methoxyindole ${ }^{\mathrm{S2}}\left((\mathrm{COCl})_{2} ; \mathrm{NH}_{4} \mathrm{OH}\left(92 \%\right.\right.$ for 2 steps); $\left.\mathrm{LiAlH}_{4}(67 \%)\right)$ or by a improved route $^{\mathrm{S3}}$ detailed below.

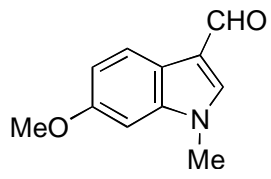

$\mathrm{POCl}_{3}\left(3.3 \mathrm{~mL}, 35.9 \mathrm{mmol}\right.$ ) was added dropwise to DMF ( $35 \mathrm{~mL}, 450 \mathrm{mmol}$ ) at $0{ }^{\circ} \mathrm{C}$. A solution of 6-methoxy-1-methylindole ${ }^{\mathrm{S} 2}(5.25 \mathrm{~g}, 32.6 \mathrm{mmol})$ in DMF $(2 \mathrm{~mL})$ was then added slowly with stirring at $0{ }^{\circ} \mathrm{C}$. The mixture was kept at $35{ }^{\circ} \mathrm{C}$ for $45 \mathrm{~min}$ and then poured onto a crushed ice. The clear solution was treated with aqueous $1 \mathrm{~N} \mathrm{NaOH}$ solution $(150 \mathrm{~mL}, 150$ $\mathrm{mmol}$ ) at $20-30{ }^{\circ} \mathrm{C}$. The last quarter was added all at once and the solution was quickly boiled for $10 \mathrm{~min}$. The cooled mixture was diluted with $\mathrm{CH}_{2} \mathrm{Cl}_{2}(100 \mathrm{~mL})$ and the organic layer was washed with water $(4 \times 50 \mathrm{~mL})$, dried over $\mathrm{MgSO}_{4}$ and evaporated under reduced pressure to afford $6.10 \mathrm{~g}(99 \%)$ of 6-methoxy-1-methylindole-3-carboxaldehyde as a light yellow oil: $\mathrm{mp}$ 124-125 ${ }^{\circ} \mathrm{C} ;{ }^{1} \mathrm{H}$ NMR $\left(500 \mathrm{MHz}, \mathrm{CDCl}_{3}\right) \delta 9.91(\mathrm{~s}, 1 \mathrm{H}), 8.24(\mathrm{~d}, J=8.8 \mathrm{~Hz}, 1 \mathrm{H}), 7.59(\mathrm{~s}, 1 \mathrm{H})$, $7.04(\mathrm{dd}, J=2.2,8.8 \mathrm{~Hz}, 1 \mathrm{H}), 6.86(\mathrm{~d}, J=2.2 \mathrm{~Hz}, 1 \mathrm{H}), 3.90(\mathrm{~s}, 3 \mathrm{H}), 3.83(\mathrm{~s}, 3 \mathrm{H})$. 


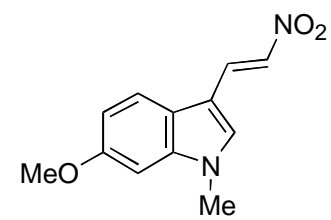

A solution of 6-methoxy-1-methylindole-3-carboxaldehyde (3.79 $\mathrm{g}, 20.0 \mathrm{mmol})$ in nitromethane $(30 \mathrm{~mL})$ was treated with ammonium acetate $(1.70 \mathrm{~g}, 22.0 \mathrm{mmol})$. The mixture was warmed at reflux under Ar for $1 \mathrm{~h}$. The reaction mixture was cooled and concentrated under reduced pressure. The residue was diluted with $\mathrm{CH}_{2} \mathrm{Cl}_{2}(100 \mathrm{~mL})$, washed with water $(2 \mathrm{x}$ $50 \mathrm{~mL}$ ) and saturated aqueous $\mathrm{NaCl}$. The organic phase was dried over $\mathrm{MgSO}_{4}$ and concentrated under reduced pressure to give a red solid, which was recrystallized (EtOAchexanes) to afford $4.60 \mathrm{~g}$ (99\%) of 6-methoxy-1-methyl-3-(2-nitroethenyl)indole as red needles: $\mathrm{mp} 138-139{ }^{\circ} \mathrm{C} ;{ }^{1} \mathrm{H}$ NMR $\left(600 \mathrm{MHz}, \mathrm{CDCl}_{3}\right) \delta 8.20(\mathrm{~d}, J=13.2 \mathrm{~Hz}, 1 \mathrm{H}), 7.74(\mathrm{~d}, J=$ $13.6 \mathrm{~Hz}, 1 \mathrm{H}), 7.66(\mathrm{~d}, J=8.8 \mathrm{~Hz}, 1 \mathrm{H}), 7.44(\mathrm{~s}, 1 \mathrm{H}), 6.99(\mathrm{dd}, J=2.2,8.8 \mathrm{~Hz}, 1 \mathrm{H}), 6.85(\mathrm{~d}, J=$ $2.2 \mathrm{~Hz}, 1 \mathrm{H}), 3.91(\mathrm{~s}, 3 \mathrm{H}), 3.85(\mathrm{~s}, 3 \mathrm{H}) ;{ }^{13} \mathrm{C} \mathrm{NMR}\left(125 \mathrm{MHz}, \mathrm{CDCl}_{3}\right) \delta 157.8,139.8,136.5$, 133.6, 131.9, 121.6, 119.6, 111.9, 108.5, 94.5, 55.9, 33.7; HRMS (MALDI-FTMS) calcd for $\left(\mathrm{C}_{11} \mathrm{H}_{11} \mathrm{~N}_{2} \mathrm{O}_{2}\right)[\mathrm{M}+\mathrm{H}]^{+}$203.0742; found 203.0742 .

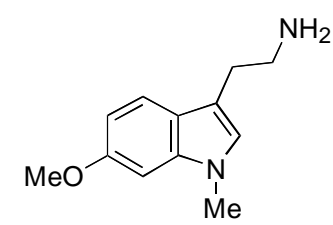

A suspension of $\mathrm{LiAlH}_{4}(2.39 \mathrm{~g}, 62.8 \mathrm{mmol})$ in anhydrous THF $(60 \mathrm{~mL})$ was treated dropwise with a solution of the 3-(2-nitroethenyl)indole $(2.43 \mathrm{~g}, 10.5 \mathrm{mmol})$ in THF $(10 \mathrm{~mL})$ at $0{ }^{\circ} \mathrm{C}$. The resulting mixture was warmed at a reflux for $2 \mathrm{~h}$. The excess hydride was destroyed by dropwise addition of saturated aqueous $\mathrm{Na}_{2} \mathrm{SO}_{4}$ and the resulting mixture was filtered through Celite. The filtrate was concentrated under reduced pressure to afford $2.04 \mathrm{~g} \mathrm{(96 \% )} \mathrm{of}$ $6^{\text {S1 }}$ as a yellow oil: ${ }^{1} \mathrm{H}$ NMR $\left(500 \mathrm{MHz}, \mathrm{CDCl}_{3}\right) \delta 7.45(\mathrm{~d}, J=8.5 \mathrm{~Hz}, 1 \mathrm{H}), 6.74-6.79(\mathrm{~m}, 3 \mathrm{H})$, $3.88(\mathrm{~s}, 3 \mathrm{H}), 3.69(\mathrm{~s}, 3 \mathrm{H}), 2.97-3.01(\mathrm{~m}, 2 \mathrm{H}), 2.83-2.87(\mathrm{~m}, 2 \mathrm{H}), 1.62(\mathrm{br} \mathrm{s}, 2 \mathrm{H})$. 


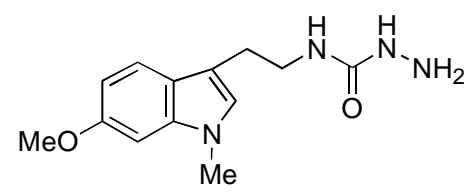

A solution of $\mathrm{N}$-methyl-6-methoxytryptamine $(6,504 \mathrm{mg}, 2.47 \mathrm{mmol}, 1.0$ equiv) dissolved in absolute EtOH $(12.3 \mathrm{~mL}$ ) was treated with diphenyl carbonate $(528 \mathrm{mg}, 2.47 \mathrm{mmol}$, 1.0 equiv) and the resulting mixture was stirred overnight at $25{ }^{\circ} \mathrm{C}$. Hydrazine monohydrate ( $0.48 \mathrm{~mL}, 9.87 \mathrm{mmol}, 4.0$ equiv) was added and the reaction mixture was warmed at reflux for $5 \mathrm{~h}$ (monitored by TLC). After cooling to $25{ }^{\circ} \mathrm{C}$, the mixture was concentrated in vacuo and absorbed onto Celite. Flash chromatography $\left(\mathrm{SiO}_{2}, 50 \%\right.$ EtOAc-hexanes, 5-10\% $\mathrm{MeOH}-\mathrm{CH}_{2} \mathrm{Cl}_{2}$ gradient elution) afforded $\mathbf{7}$ as a tan solid. This solid was dissolved in minimal $\mathrm{CH}_{2} \mathrm{Cl}_{2}$ and $\mathrm{Et}_{2} \mathrm{O}$ was added until a free floating white solid precipitated with stirring to provide pure 7 (369 mg, $64 \%):{ }^{1} \mathrm{H}$ NMR $\left(600 \mathrm{MHz}, \mathrm{CDCl}_{3}\right) \delta 7.48(\mathrm{~d}, J=8.6 \mathrm{~Hz}, 1 \mathrm{H}), 6.74-6.80(\mathrm{~m}, 3 \mathrm{H}), 6.08(\mathrm{br} \mathrm{s}, 1 \mathrm{H})$, $5.77(\mathrm{br} \mathrm{s}, 1 \mathrm{H}), 3.88(\mathrm{~s}, 3 \mathrm{H}), 3.69(\mathrm{~s}, 3 \mathrm{H}), 3.54(\mathrm{t}, J=6.6 \mathrm{~Hz}, 2 \mathrm{H}), 2.93(\mathrm{t}, J=6.8 \mathrm{~Hz}, 2 \mathrm{H}) ;{ }^{13} \mathrm{C}$ NMR $\left(125 \mathrm{MHz}, \mathrm{C}_{6} \mathrm{D}_{6}\right) \delta 163.1,160.1,156.4,137.7,125.5,122.4,119.6,112.0,108.7,92.8$, 55.7, 40.2, 32.6, 26.0; IR (film) 3350, 2931, 1619, 1534, 1472, 1431, 1378, 1325, 1244, 1220, $1161,1032,791 \mathrm{~cm}^{-1}$; HRMS (MALDI-FTMS) calcd for $\left(\mathrm{C}_{13} \mathrm{H}_{18} \mathrm{~N}_{4} \mathrm{O}_{2}\right)[\mathrm{M}+\mathrm{H}]^{+} 263.1502$; found 263.1493.

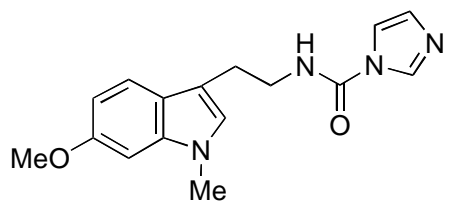

N-Methyl-6-methoxytryptamine $(6,6.91 \mathrm{~g}, 34.2 \mathrm{mmol})$ in anhydrous $\mathrm{CH}_{2} \mathrm{Cl}_{2}(40 \mathrm{~mL})$ was added dropwise to a solution of 1,1-carbonyldiimidazole (CDI, $11.08 \mathrm{~g}, 68.3 \mathrm{mmol}$ ) in anhydrous THF ( $40 \mathrm{~mL})$ under $\operatorname{Ar}$ at $0{ }^{\circ} \mathrm{C}$. The reaction mixture was allowed to warm to $25{ }^{\circ} \mathrm{C}$ overnight. Upon completion of the reaction as monitored by TLC, the reaction mixture was concentrated onto Celite in vacuo. Flash chromatography $\left(\mathrm{SiO}_{2}, 5 \% \mathrm{MeOH}-\mathrm{CHCl}_{3}\right)$ afforded 8 as a pale yellow foam $(9.17 \mathrm{~g}, 90 \%):{ }^{1} \mathrm{H}$ NMR $\left(500 \mathrm{MHz}, \mathrm{CDCl}_{3}\right) \delta 7.94(\mathrm{~s}, 1 \mathrm{H}), 7.42(\mathrm{~d}, J=8.4$ $\mathrm{Hz}, 1 \mathrm{H}), 7.22(\mathrm{~s}, 1 \mathrm{H}), 6.98(\mathrm{~s}, 1 \mathrm{H}), 6.75-6.79(\mathrm{~m}, 3 \mathrm{H}), 6.32(\mathrm{br} \mathrm{s}, 1 \mathrm{H}), 3.87(\mathrm{~s}, 3 \mathrm{H}), 3.66-3.72$ $(\mathrm{m}, 5 \mathrm{H}), 3.03(\mathrm{t}, J=6.6 \mathrm{~Hz}, 2 \mathrm{H}) ;{ }^{13} \mathrm{C}$ NMR $\left(125 \mathrm{MHz}, \mathrm{C}_{6} \mathrm{D}_{6}\right) \delta 156.6,148.8,137.9,135.7$, $130.2,125.8,122.0,119.2,115.9,110.8,109.2,93.0,55.7,41.6,32.7,25.0$. 


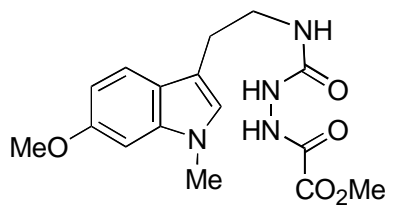

Conversion of 7 to 9. A solution of 7 (119 mg, $0.51 \mathrm{mmol}, 1.0$ equiv) and oxalic acid mono-methyl ester ( $80 \mathrm{mg}, 0.77 \mathrm{mmol}, 1.5$ equiv) in anhydrous $\mathrm{CH}_{2} \mathrm{Cl}_{2}(3 \mathrm{~mL})$ cooled to $0{ }^{\circ} \mathrm{C}$ was treated with EDCI (147 mg, $0.77 \mathrm{mmol}, 1.5$ equiv). After $5 \mathrm{~min}$, DMAP (94 mg, $0.77 \mathrm{mmol}$, 1.5 equiv) was added and the resulting reaction mixture was stirred at $25{ }^{\circ} \mathrm{C}$ overnight. The mixture was concentrated in vacuo and absorbed onto Celite. Flash chromatography $\left(\mathrm{SiO}_{2}, 1-\right.$ $5 \% \mathrm{MeOH}-\mathrm{CHCl}_{3}$ ) afforded 9 as a yellow/tan colored solid (96 mg, 61\%).

Conversion of 8 to 9. A solution of $8(2.22 \mathrm{~g}, 7.44 \mathrm{mmol})$ and $\mathrm{H}_{2} \mathrm{NHNCOCO}_{2} \mathrm{Me}(879$ $\mathrm{mg}, 7.44 \mathrm{mmol})$ in THF ( $40 \mathrm{~mL})$ at $35^{\circ} \mathrm{C}$ was treated with glacial $\mathrm{ACOH}(2 \mathrm{~mL})$ and the resulting mixture was stirred at $35{ }^{\circ} \mathrm{C}$ overnight. After approximately $19 \mathrm{~h}$, the reaction mixture was concentrated onto Celite. Flash chromatography $\left(\mathrm{SiO}_{2}, 5: 70: 25 \mathrm{MeOH}-\mathrm{CHCl}_{3}\right.$-acetone) afforded 9 as a white solid $(2.05 \mathrm{~g}, 79 \%)$ : $\mathrm{mp} 162-163{ }^{\circ} \mathrm{C} ;{ }^{1} \mathrm{H} \mathrm{NMR}\left(400 \mathrm{MHz}, \mathrm{CDCl}_{3}\right) \delta 9.49$ (br s, $\left.1 \mathrm{H}\right)$, 7.88 (br s, 1H), $7.38(\mathrm{~d}, J=8.5 \mathrm{~Hz}, 1 \mathrm{H}), 6.68-6.77(\mathrm{~m}, 3 \mathrm{H}), 5.76(\mathrm{br} \mathrm{s}, 1 \mathrm{H}), 3.85(\mathrm{~s}, 3 \mathrm{H}), 3.79$ $(\mathrm{s}, 3 \mathrm{H}), 3.60(\mathrm{~s}, 3 \mathrm{H}), 3.40-3.43(\mathrm{~m}, 2 \mathrm{H}), 2.83(\mathrm{t}, J=6.6 \mathrm{~Hz}, 2 \mathrm{H}) ;{ }^{13} \mathrm{C} \mathrm{NMR}\left(100 \mathrm{MHz}, \mathrm{C}_{6} \mathrm{D}_{6}\right)$ $\delta$ 159.2, 157.1, 156.3, 154.8, 137.7, 125.9, 122.1, 119.4, 111.2, 108.7, 92.8, 55.7, 53.6, 40.7, 32.5, 25.5; IR (film) 3348, 2938, 1721, 1648, 1560, 1482, 1439, 1212, 1118, 1070, $806 \mathrm{~cm}^{-1}$; HRMS (MALDI-FTMS) calcd for $\left(\mathrm{C}_{16} \mathrm{H}_{21} \mathrm{~N}_{4} \mathrm{O}_{5}\right)[\mathrm{M}+\mathrm{H}]^{+} 349.1506$; found 349.1491 .

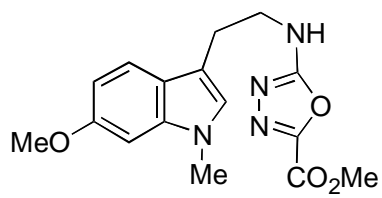

A solution of $9(3.27 \mathrm{~g}, 9.38 \mathrm{mmol})$ in $\mathrm{CH}_{2} \mathrm{Cl}_{2}(3 \mathrm{~mL})$ at $0{ }^{\circ} \mathrm{C}$ was treated with $p$ toluenesulfonyl chloride $\left(1.79 \mathrm{~g}, 9.38 \mathrm{mmol}\right.$ ) followed by $\mathrm{Et}_{3} \mathrm{~N}(3.25 \mathrm{~mL}, 23.45 \mathrm{mmol})$. The resulting reaction mixture was stirred overnight at $25{ }^{\circ} \mathrm{C}$. After $16-20 \mathrm{~h}$, the solvent was removed under reduced pressure and the material was absorbed onto Celite. Flash chromatography $\left(\mathrm{SiO}_{2}, 50 \%\right.$ EtOAc-hexanes) afforded 10 as a white solid $(2.08 \mathrm{~g}, 67 \%): \mathrm{mp}$ 156-157 ${ }^{\circ} \mathrm{C} ;{ }^{1} \mathrm{H}$ NMR $\left(400 \mathrm{MHz}, \mathrm{CDCl}_{3}\right) \delta 7.43(\mathrm{~d}, J=8.6 \mathrm{~Hz}, 1 \mathrm{H}), 6.75-6.81(\mathrm{~m}, 3 \mathrm{H}), 5.28(\mathrm{br}$ $\mathrm{s}, 1 \mathrm{H}), 3.98(\mathrm{~s}, 3 \mathrm{H}), 3.88(\mathrm{~s}, 3 \mathrm{H}), 3.74(\mathrm{dd}, J=6.3,12.5 \mathrm{~Hz}, 2 \mathrm{H}), 3.70(\mathrm{~s}, 3 \mathrm{H}), 3.07(\mathrm{t}, J=6.5$ 
$\mathrm{Hz}, 2 \mathrm{H}) ;{ }^{13} \mathrm{C}$ NMR $\left(100 \mathrm{MHz}, \mathrm{C}_{6} \mathrm{D}_{6}\right) \delta 164.5,156.6,154.8,150.9,137.9,126.0,121.7,119.3$, 110.0, 109.1, 92.9, 55.7, 53.2, 43.5, 32.6, 25.1; IR (film) 3350, 2939, 1740, 1625, 1551, 1438, $1384,1254,1152,814 \mathrm{~cm}^{-1}$; HRMS (MALDI-FTMS) calcd for $\left(\mathrm{C}_{16} \mathrm{H}_{20} \mathrm{~N}_{4} \mathrm{O}_{4}\right)[\mathrm{M}+\mathrm{H}]^{+} 333.1557$; found 333.1563 .

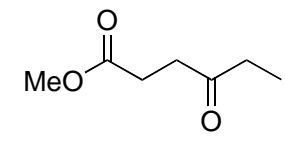

Succinyl dichloride $(4.6 \mathrm{~mL}, 41.6 \mathrm{mmol})$ in $\mathrm{CH}_{2} \mathrm{Cl}_{2}(200 \mathrm{~mL})$ was cooled to $-40{ }^{\circ} \mathrm{C}$ and ethylaluminum dichloride $(50 \mathrm{~mL}, 50 \mathrm{mmol})$ was added at $-40{ }^{\circ} \mathrm{C}$. The reaction mixture was stirred at $-40{ }^{\circ} \mathrm{C}$ for $3.5 \mathrm{~h}$ and quenched with the addition of anhydrous $\mathrm{MeOH}(200 \mathrm{~mL})$. The reaction mixture was concentrated to dryness and redissolved in $\mathrm{CH}_{2} \mathrm{Cl}_{2}(100 \mathrm{~mL})$. The organic layer was washed with aqueous sodium potassium tartrate $(2 \times 50 \mathrm{~mL})$, water $(50 \mathrm{~mL})$ and saturated aqueous $\mathrm{NaCl}(50 \mathrm{~mL})$. The organic layer was dried over $\mathrm{MgSO}_{4}$, filtered and concentrated to provide pure methyl 4-ketohexanoate ${ }^{54}(6.0 \mathrm{~g}, 99 \%):{ }^{1} \mathrm{H}$ NMR $(400 \mathrm{MHz}$, $\left.\mathrm{CDCl}_{3}\right) \delta 3.67(\mathrm{~s}, 3 \mathrm{H}), 2.74(\mathrm{t}, J=6.5 \mathrm{~Hz}, 2 \mathrm{H}), 2.60(\mathrm{t}, J=6.5 \mathrm{~Hz}, 2 \mathrm{H}), 2.51(\mathrm{q}, J=7.3 \mathrm{~Hz}$, 2H), $1.09(\mathrm{~J}=7.3 \mathrm{~Hz}, 3 \mathrm{H}) ;{ }^{13} \mathrm{C} \mathrm{NMR}\left(100 \mathrm{MHz}, \mathrm{CDCl}_{3}\right) \delta 209.4,173.3,51.7,36.6,35.9,27.7$, 7.7 .

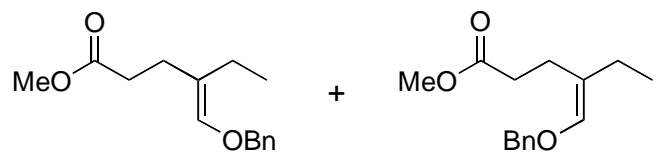

$(1: 1)$

A suspension of (benzyloxymethyl)triphenylphosphonium bromide $(83.8 \mathrm{~g}, 200 \mathrm{mmol}$ ) in anhydrous THF (120 mL) was cooled to $-78{ }^{\circ} \mathrm{C}$ and treated dropwise with NaHMDS (100 mL, $200 \mathrm{mmol}$ ) in THF. Upon final addition of NaHMDS, the reaction mixture was warmed to $-40{ }^{\circ} \mathrm{C}$ and was stirred for 30-40 min (mixture turns a deep red color). The mixture was recooled to $78{ }^{\circ} \mathrm{C}$ and a solution of methyl 4-ketohexanoate $(12.6 \mathrm{~g}, 100 \mathrm{mmol})$ in THF $(20 \mathrm{~mL})$ was added slowly via a cannula. The resulting mixture was stirred overnight while slowly warming to $25^{\circ} \mathrm{C}$. The reaction mixture was quenched by addition of $1 \mathrm{~N}$ aqueous $\mathrm{NaH}_{2} \mathrm{PO}_{4}(100 \mathrm{~mL})$, extracted with $\mathrm{Et}_{2} \mathrm{O}(3 \times 100 \mathrm{~mL})$, and the combined organic layers were dried over $\mathrm{MgSO}_{4}$, filtered, and concentrated in vacuo. The crude material (ca $1: 1$ mixture of isomers $(23.6 \mathrm{~g}, 86 \%)$ ) obtained was purified by flash chromatography $\left(\mathrm{SiO}_{2}, 2-4 \%\right.$ EtOAc-hexanes) to afford a clear oil. The $(Z)$-isomer eluted first off the column $(10.3 \mathrm{~g})$, followed by the $(E)$-isomer $(11.0 \mathrm{~g})$. 
(E)-enol ether: ${ }^{1} \mathrm{H}$ NMR $\left(600 \mathrm{MHz}, \mathrm{CDCl}_{3}\right) \delta 7.30-7.35(\mathrm{~m}, 5 \mathrm{H}), 5.90(\mathrm{~s}, 1 \mathrm{H}), 4.73(\mathrm{~s}$, $2 \mathrm{H}), 3.61(\mathrm{~s}, 3 \mathrm{H}), 2.36(\mathrm{t}, J=7.9 \mathrm{~Hz}, 2 \mathrm{H}), 2.21(\mathrm{t}, J=7.9 \mathrm{~Hz}, 2 \mathrm{H}), 2.12(\mathrm{q}, J=7.4 \mathrm{~Hz}, 2 \mathrm{H})$, $0.97(\mathrm{t}, J=7.4 \mathrm{~Hz}, 3 \mathrm{H}) ;{ }^{13} \mathrm{C}$ NMR $\left(150 \mathrm{MHz}, \mathrm{C}_{6} \mathrm{D}_{6}\right) \delta 173.7,140.7,137.8,128.3(2 \mathrm{C}), 127.7$, 127.2 (2C), 118.9, 73.4, 51.4, 33.3, 26.7, 20.0, 12.5; HRMS (MALDI-FTMS) calcd for $\left(\mathrm{C}_{15} \mathrm{H}_{20} \mathrm{O}_{3}\right)$ $[\mathrm{M}+\mathrm{Na}]^{+}$271.1305; found 271.1308.

(Z)-enol ether: ${ }^{1} \mathrm{H}$ NMR $\left(500 \mathrm{MHz}, \mathrm{CDCl}_{3}\right) \delta 7.27-7.38(\mathrm{~m}, 5 \mathrm{H}), 5.92(\mathrm{~s}, 1 \mathrm{H}), 4.74(\mathrm{~s}$, 2H), $3.64(\mathrm{~s}, 3 \mathrm{H}), 2.36-2.47(\mathrm{~m}, 4 \mathrm{H}), 1.90(\mathrm{q}, J=7.4 \mathrm{~Hz}, 2 \mathrm{H}), 0.96(\mathrm{t}, J=7.5 \mathrm{~Hz}, 3 \mathrm{H}) ;{ }^{13} \mathrm{C}$ NMR $\left(125 \mathrm{MHz}, \mathrm{C}_{6} \mathrm{D}_{6}\right) \delta 174.1,140.7,137.8,128.4$ (2C), 127.7, 127.3 (2C), 118.8, 73.4, 51.4, $32.6,24.8,23.0,13.1$.

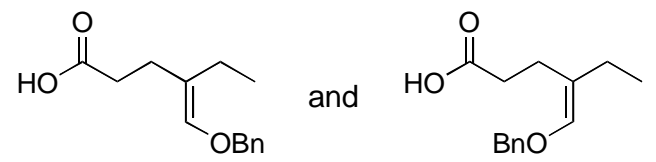

A solution of methyl (Z)-5-benzyloxy-4-ethylpent-4-enoate $(3.0 \mathrm{~g}, 12 \mathrm{mmol})$ in a $3: 1$ mixture of THF (18 mL):MeOH ( $6 \mathrm{~mL}$ ) was cooled to $0{ }^{\circ} \mathrm{C}$ and $1 \mathrm{~N}$ aqueous $\mathrm{LiOH}(36 \mathrm{~mL}, 36$ mmol) was added. The mixture was stirred at $0-25{ }^{\circ} \mathrm{C}$ (ca $3 \mathrm{~h}$ ) prior to dilution with $\mathrm{H}_{2} \mathrm{O}$, ethyl acetate and addition of $10 \%$ aqueous citric acid until the mixture reached $\mathrm{pH} 3-4$. The cloudy solution was extracted with EtOAc $(3 \times 50 \mathrm{~mL})$, and the combined organic layers were washed with $\mathrm{H}_{2} \mathrm{O}$ and saturated aqueous $\mathrm{NaCl}$, dried over $\mathrm{Na}_{2} \mathrm{SO}_{4}$, filtered, and concentrated in vacuo to afford ( $Z$-11 as a clear oil $(2.6 \mathrm{~g}, 93 \%)$. If necessary, this oil can be further purified by flash chromatography ( $\mathrm{SiO}_{2}, 25 \%$ EtOAc-hexanes): ${ }^{1} \mathrm{H} \mathrm{NMR}\left(500 \mathrm{MHz}, \mathrm{CDCl}_{3}\right) \delta 7.28-7.37(\mathrm{~m}, 5 \mathrm{H})$, $5.93(\mathrm{~s}, 1 \mathrm{H}), 4.74(\mathrm{~s}, 2 \mathrm{H}), 2.40-2.47(\mathrm{~m}, 4 \mathrm{H}), 1.91(\mathrm{dd}, J=7.4,15.0 \mathrm{~Hz}, 2 \mathrm{H}), 0.98(\mathrm{t}, J=7.3$ $\mathrm{Hz}, 3 \mathrm{H}) ;{ }^{13} \mathrm{C}$ NMR $\left(125 \mathrm{MHz}, \mathrm{C}_{6} \mathrm{D}_{6}\right) \delta 179.3,140.9,137.8,128.4(2 \mathrm{C}), 127.7,127.3(2 \mathrm{C}), 118.6$, $73.5,32.5,24.8,22.8,13.1$; HRMS (MALDI-FTMS) calcd for $\left(\mathrm{C}_{14} \mathrm{H}_{18} \mathrm{O}_{3}\right)[\mathrm{M}+\mathrm{Na}]^{+} 257.1149$; found 257.1149 .

Similarly, hydrolysis of the $(E)$-enol ether afforded $(E)-\mathbf{1 1}:{ }^{1} \mathrm{H} \mathrm{NMR}\left(500 \mathrm{MHz}, \mathrm{CDCl}_{3}\right) \delta$ 7.27-7.38 (m, 5H), $5.93(\mathrm{~s}, 1 \mathrm{H}), 4.75(\mathrm{~s}, 2 \mathrm{H}), 2.41(\mathrm{t}, J=7.7 \mathrm{~Hz}, 2 \mathrm{H}), 2.23(\mathrm{t}, J=7.7 \mathrm{~Hz}, 2 \mathrm{H})$, $2.13(\mathrm{dd}, J=7.7,15.0 \mathrm{~Hz}, 2 \mathrm{H}), 0.98(\mathrm{t}, J=7.5 \mathrm{~Hz}, 3 \mathrm{H}) ;{ }^{13} \mathrm{C}$ NMR $\left(125 \mathrm{MHz}, \mathrm{C}_{6} \mathrm{D}_{6}\right) \delta 179.5$, $140.8,137.8,128.4$ (2C), 127.7, 127.3 (2C), 118.7, 73.5, 33.3, 26.4, 20.0, 12.5; HRMS (MALDI-FTMS) calcd for $\left(\mathrm{C}_{14} \mathrm{H}_{18} \mathrm{O}_{3}\right)[\mathrm{M}+\mathrm{Na}]^{+} 257.1149$; found 257.1149 . 


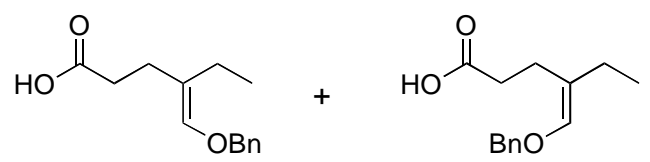

$(85: 15)$

( $E$ - and (Z)-5-Benzyloxy-4-ethylpent-4-enoic acid: direct Wittig reaction. A solution of (benzyloxymethyl)triphenylphosphonium bromide $(5.00 \mathrm{~g}, 11.9 \mathrm{mmol}$ ) in anhydrous THF (52 $\mathrm{mL}$ ) was cooled to $-78{ }^{\circ} \mathrm{C}$ and treated dropwise with $t$-BuLi $(5.9 \mathrm{~mL}, 10.0 \mathrm{mmol}, 1.7 \mathrm{M}$ solution in pentane). The reaction mixture was stirred for $5 \mathrm{~min}$ at $-78{ }^{\circ} \mathrm{C}$ before warming to $-40{ }^{\circ} \mathrm{C}$ where the solution was stirred for 30-40 min (mixture turns a deep red color), and then recooled to $-78{ }^{\circ} \mathrm{C}$. A solution of 4-ketohexanoic acid (260 mg, $2.0 \mathrm{mmol}$ ) in THF (3 mL) was added slowly via a cannula and the resulting mixture was stirred overnight while slowly warming to $25{ }^{\circ} \mathrm{C}$. The reaction mixture was quenched by careful addition of $1 \mathrm{~N}$ aqueous $\mathrm{NaH}_{2} \mathrm{PO}_{4}(52 \mathrm{~mL})$, extracted with $\mathrm{Et}_{2} \mathrm{O}(3 \times 75 \mathrm{~mL})$, and the combined organic layers were dried over $\mathrm{Na}_{2} \mathrm{SO}_{4}$, filtered, and concentrated in vacuo. The crude material (ca. 85:15 ratio of isomers by GC) thus obtained was purified by flash chromatography $\left(\mathrm{SiO}_{2}, 10-100 \%\right.$ EtOAc-hexanes gradient elution) to afford a yellow oil (262 mg, 56\%) which was found to be almost exclusively one enol ether by GC and ${ }^{1} \mathrm{H}$ NMR (NOE) after chromatography. Continued purification can furnish $>95 \%$ pure $(E)$ - as well as $>95 \%(Z)$-enol ether as separate fractions, as determined by the respective GC traces of the individual fractions.

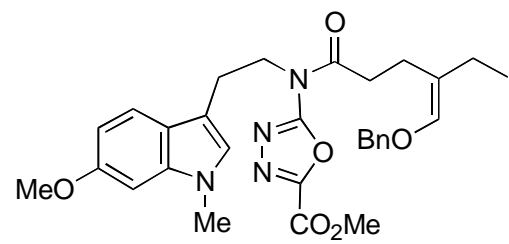

Oxadiazole 10 (345 mg, $1.04 \mathrm{mmol}$ ) was added to a solution of (Z)-11 (>97\% Z-enol ether by GC analysis, $1.30 \mathrm{~g}, 5.55 \mathrm{mmol})$ in anhydrous $\mathrm{CH}_{2} \mathrm{Cl}_{2}(150 \mathrm{~mL})$ at $0{ }^{\circ} \mathrm{C}$ under Ar. The mixture was cooled to $0{ }^{\circ} \mathrm{C}$ and EDCI ( $854 \mathrm{mg}, 4.45 \mathrm{mmol}$ ) was added. The resulting mixture was subsequently stirred for $2 \mathrm{~min}$, before the addition of DMAP (550 mg, $4.50 \mathrm{mmol}$ ). The reaction mixture was stirred $(16 \mathrm{~h})$ while slowly warming to $25{ }^{\circ} \mathrm{C}$, at which time the solvent was removed under reduced pressure absorbing the mixture onto Celite. Flash chromatography $\left(\mathrm{SiO}_{2}, 30 \%\right.$ EtOAc-hexanes) afforded $4 \mathrm{a}(550 \mathrm{mg}, 96 \%)$ as a white solid: $\mathrm{mp} 86-87{ }^{\circ} \mathrm{C} ;{ }^{1} \mathrm{H}$ $\operatorname{NMR}\left(600 \mathrm{MHz}, \mathrm{CDCl}_{3}\right) \delta 7.54(\mathrm{~d}, J=8.3 \mathrm{~Hz}, 1 \mathrm{H}), 7.24-7.33(\mathrm{~m}, 5 \mathrm{H}), 6.78(\mathrm{dd}, J=2.2,8.8 \mathrm{~Hz}$, 
$1 \mathrm{H}), 6.72(\mathrm{~s}, 1 \mathrm{H}), 6.68(\mathrm{~d}, J=6.9 \mathrm{~Hz}, 1 \mathrm{H}), 5.90(\mathrm{~s}, 1 \mathrm{H}), 4.70(\mathrm{~s}, 2 \mathrm{H}), 4.17(\mathrm{t}, J=7.7 \mathrm{~Hz}, 2 \mathrm{H})$, $3.98(\mathrm{~s}, 3 \mathrm{H}), 3.87(\mathrm{~s}, 3 \mathrm{H}), 3.64(\mathrm{~s}, 3 \mathrm{H}), 3.05(\mathrm{t}, J=7.7 \mathrm{~Hz}, 2 \mathrm{H}), 2.91(\mathrm{t}, J=7.7 \mathrm{~Hz}, 2 \mathrm{H}), 2.45$ $(\mathrm{t}, J=7.7 \mathrm{~Hz}, 2 \mathrm{H}), 1.89(\mathrm{q}, J=7.4 \mathrm{~Hz}, 2 \mathrm{H}), 0.95(\mathrm{t}, J=7.4 \mathrm{~Hz}, 3 \mathrm{H}) ;{ }^{1} \mathrm{H} \mathrm{NMR}\left(600 \mathrm{MHz}, \mathrm{C}_{6} \mathrm{D}_{6}\right)$ $\delta 7.78(\mathrm{~d}, J=8.6 \mathrm{~Hz}, 1 \mathrm{H}), 7.12-7.20(\mathrm{~m}, 3 \mathrm{H}), 7.00-7.04(\mathrm{~m}, 2 \mathrm{H}), 6.58(\mathrm{dd}, J=1.7 \mathrm{~Hz}, 1 \mathrm{H})$, $6.25(\mathrm{~s}, 1 \mathrm{H}), 5.79(\mathrm{~s}, 1 \mathrm{H}), 4.42(\mathrm{~s}, 2 \mathrm{H}), 4.10(\mathrm{t}, J=7.7 \mathrm{~Hz}, 2 \mathrm{H}), 3.51(\mathrm{~s}, 3 \mathrm{H}), 3.33(\mathrm{~s}, 3 \mathrm{H}), 3.11$ $(\mathrm{t}, J=7.7 \mathrm{~Hz}, 2 \mathrm{H}), 3.03(\mathrm{t}, J=7.7 \mathrm{~Hz}, 2 \mathrm{H}), 2.93(\mathrm{~s}, 3 \mathrm{H}), 2.75(\mathrm{t}, J=7.7 \mathrm{~Hz}, 2 \mathrm{H}), 1.89(\mathrm{q}, J=$ $7.2 \mathrm{~Hz}, 2 \mathrm{H}), 0.94(\mathrm{t}, J=7.4 \mathrm{~Hz}, 3 \mathrm{H}) ;{ }^{13} \mathrm{C}$ NMR $\left(100 \mathrm{MHz}, \mathrm{CDCl}_{3}\right) \delta 172.4,162.1,156.4,154.1$, $153.1,140.9,137.7,137.6,128.4$ (2C) $127.7,127.3$ (2C), 126.4, 121.7, 119.6, 118.5, 110.0, 109.0, 92.7, 73.4, 55.6, 53.5, 47.7, 34.6, 32.5, 24.9, 24.4, 23.0, $13.1{ }_{1}^{13} \mathrm{C} N M R\left(100 \mathrm{MHz}, \mathrm{C}_{6} \mathrm{D}_{6}\right)$ $\delta 172.0,162.3,157.1,154.4,153.2,141.4,138.3,128.6,128.3$ (2C), 128.1, 127.9, 127.5 (2C), $126.4,122.6,120.2,118.7,110.5,109.3,93.2,73.5,55.2,52.5,47.7,35.4,31.8,25.4,24.7$, 23.6, 13.4; IR (film) 3446, 2943, 1749, 1703, 1626, 1564, 1441, 1262, 1226, $1154 \mathrm{~cm}^{-1}$; HRMS (MALDI-FTMS) calcd for $\left(\mathrm{C}_{30} \mathrm{H}_{34} \mathrm{~N}_{4} \mathrm{O}_{6}\right)[\mathrm{M}+\mathrm{H}]^{+} 547.2551$; found 547.2554 .

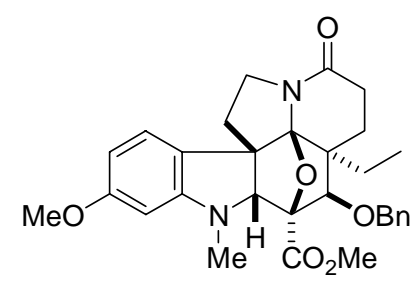

A solution of $4 \mathrm{a}(3 \times 10.9 \mathrm{mg}, 0.060 \mathrm{mmol})$ in triisopropylbenzene $(3 \times 200 \mathrm{~mL}, 0.1 \mathrm{mM})$ was purged with Ar for 30 min and was warmed at $230{ }^{\circ} \mathrm{C}$ for $90 \mathrm{~h}$. The reaction mixture was cooled to room temperature, and loaded directly onto a silica gel chromatography column. The triisopropylbenzene was eluted with hexanes, and then the column was eluted with $50 \%$ EtOAc-hexanes to afford $\mathbf{5 a}(15.4 \mathrm{mg}, 50 \%)$ as a yellow foam. Alternatively, a solution of $\mathbf{4 a}$ (2 $\times 4.3 \mathrm{mg}, 0.016 \mathrm{mmol}$ ) in triisopropylbenzene $(2 \times 160 \mathrm{~mL}, 0.05 \mathrm{mM}$ ) was purged with Ar for $30 \mathrm{~min}$ and was warmed at $230{ }^{\circ} \mathrm{C}$ for $90 \mathrm{~h}$. The reaction mixture was cooled to room temperature, and loaded directly onto a silica gel chromatography column. The triisopropylbenzene was eluted with hexanes, and then the column was eluted with $50 \%$ EtOAc-hexanes to afford $\mathbf{5 a}\left(4.2 \mathrm{mg}, 53 \%\right.$ ) as a yellow foam: $\mathrm{mp} 203-205{ }^{\circ} \mathrm{C}$ (EtOAchexanes); ${ }^{1} \mathrm{H}$ NMR (500 MHz, $\left.\mathrm{C}_{6} \mathrm{D}_{6}\right) \delta 7.26(\mathrm{~d}, J=7.3 \mathrm{~Hz}, 2 \mathrm{H}), 7.07-7.18(\mathrm{~m}, 3 \mathrm{H}), 6.51(\mathrm{~d}, J=$ $8.1 \mathrm{~Hz}, 1 \mathrm{H}), 6.18(\mathrm{dd}, J=2.2,8.1 \mathrm{~Hz}, 1 \mathrm{H}), 6.10(\mathrm{~d}, J=2.2 \mathrm{~Hz}, 1 \mathrm{H}), 4.51(\mathrm{~d}, J=11.0 \mathrm{~Hz}, 1 \mathrm{H})$, $4.33(\mathrm{~d}, J=11.0 \mathrm{~Hz}, 1 \mathrm{H}), 4.31(\mathrm{~s}, 1 \mathrm{H}), 3.88-3.97(\mathrm{~m}, 2 \mathrm{H}), 3.80(\mathrm{~s}, 1 \mathrm{H}), 3.40(\mathrm{~s}, 3 \mathrm{H}), 3.38(\mathrm{~s}$, $3 \mathrm{H}), 2.87(\mathrm{~s}, 3 \mathrm{H}), 2.60(\mathrm{dd}, J=5.9,13.9 \mathrm{~Hz}, 1 \mathrm{H}), 2.32(\mathrm{dd}, J=4.6,18.5 \mathrm{~Hz}, 1 \mathrm{H}), 2.07-2.19$ 
$(\mathrm{m}, 2 \mathrm{H}), 1.69$ (ddd, $J=3.7,5.5,12.5 \mathrm{~Hz}, 1 \mathrm{H}), 1.45(\mathrm{dd}, J=4.8,14.3 \mathrm{~Hz}, 1 \mathrm{H}), 0.89-0.98(\mathrm{~m}$, $1 \mathrm{H}), 0.68(\mathrm{dq}, J=7.3,14.7 \mathrm{~Hz}, 1 \mathrm{H}), 0.42(\mathrm{t}, J=7.3 \mathrm{~Hz}, 3 \mathrm{H}) ;{ }^{13} \mathrm{C} \mathrm{NMR}\left(125 \mathrm{MHz}, \mathrm{C}_{6} \mathrm{D}_{6}\right)$ $\delta 170.6,169.6,162.3,155.1,138.8,129.0$ (2C), 128.1 (2C), 124.5, 122.0, 105.0, 103.6, 95.1, $91.5,87.6,82.3,77.9,74.6,64.5,55.1,51.8,50.3,47.4,38.0,35.6,28.9,22.9,21.7,10.1$; IR (neat) 3444, 2953, 1734, 1664, 1620,1493, 1400, 1247, $1097 \mathrm{~cm}^{-1}$; HRMS (MALDI-FTMS) calcd for $\left(\mathrm{C}_{30} \mathrm{H}_{35} \mathrm{~N}_{2} \mathrm{O}_{6}\right)[\mathrm{M}+\mathrm{H}]^{+} 519.2490$; found 519.2498. A sample of 5a (CCDC 253429) for $\mathrm{X}$-ray analysis was obtained by recrystallization from EtOAc-hexanes: $\mathrm{mp} 203-205{ }^{\circ} \mathrm{C}$. The enantiomers of $5 \mathbf{a}(40 \mathrm{mg} /$ injection) were separated $(\alpha=1.70)$ on a semipreparative Chiralcel OD column $(2 \times 25 \mathrm{~cm}, 30 \%$ PPrOH-hexanes, $10 \mathrm{~mL} / \mathrm{min}$ flow rate) providing natural-(+)-5a $\left(t_{R}=15.1 \mathrm{~min}\right)$ and ent $(-)-5 \mathbf{a}\left(t_{\mathrm{R}}=25.6 \mathrm{~min}\right)$. For natural-(+)-5a: $[\alpha]_{\mathrm{D}}^{23}+50(c 1.4, \mathrm{MeOH}) ;$ for ent-(-)-5a: $[\alpha]_{\mathrm{D}}^{23}-49(c 2.1, \mathrm{MeOH})$.

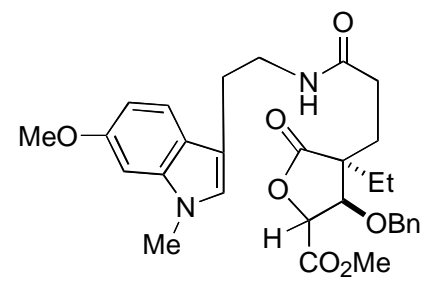

Data for lactone isolated upon premature workup of the cycloaddition of $4 \mathrm{a}:{ }^{1} \mathrm{H}$ NMR $\left(500 \mathrm{MHz}, \mathrm{C}_{6} \mathrm{D}_{6}\right) \delta 7.50(\mathrm{~d}, J=8.4 \mathrm{~Hz}, 1 \mathrm{H}), 6.97-7.21(\mathrm{~m}, 6 \mathrm{H}), 6.64(\mathrm{~d}, J=1.8 \mathrm{~Hz}, 1 \mathrm{H}), 6.34$ $(\mathrm{s}, 1 \mathrm{H}), 4.67-4.72(\mathrm{~m}, 1 \mathrm{H}), 4.49(\mathrm{~d}, J=5.1 \mathrm{~Hz}, 1 \mathrm{H}), 4.38(\mathrm{~d}, J=11.7 \mathrm{~Hz}, 1 \mathrm{H}), 4.25(\mathrm{~d}, J=$ $11.7 \mathrm{~Hz}, 1 \mathrm{H}), 4.09(\mathrm{~d}, J=4.8 \mathrm{~Hz}, 1 \mathrm{H}), 3.55(\mathrm{~s}, 3 \mathrm{H}), 3.44(\mathrm{dt}, J=2.6,6.6 \mathrm{~Hz}, 2 \mathrm{H}), 3.19(\mathrm{~s}, 3 \mathrm{H})$, $2.96(\mathrm{~s}, 3 \mathrm{H}), 2.79(\mathrm{t}, J=7.0 \mathrm{~Hz}, 2 \mathrm{H}), 2.28-2.48(\mathrm{~m}, 3 \mathrm{H}), 2.12-2.19(\mathrm{~m}, 1 \mathrm{H}), 1.21-1.51(\mathrm{~m}$, $2 \mathrm{H}), 0.69(\mathrm{t}, J=7.3 \mathrm{~Hz}, 3 \mathrm{H}) ;{ }^{13} \mathrm{C}$ NMR $\left(125 \mathrm{MHz}, \mathrm{C}_{6} \mathrm{D}_{6}\right) \delta 176.8,171.0,169.5,157.2,138.3$, 137.2, 129.0 (2C), 128.1 (2C), 125.5, 123.0, 119.9, 112.1, 109.1, 93.2, 81.7, 78.6, 72.4, 55.2, 52.0, 49.9, 40.2, 31.8, 31.4, 30.1, 27.6, 26.7, 25.7, 8.2; HRMS (MALDI-FTMS) calcd for $\left(\mathrm{C}_{30} \mathrm{H}_{37} \mathrm{~N}_{2} \mathrm{O}_{7}\right)[\mathrm{M}+\mathrm{H}]^{+}$537.2523; found 537.2596 .

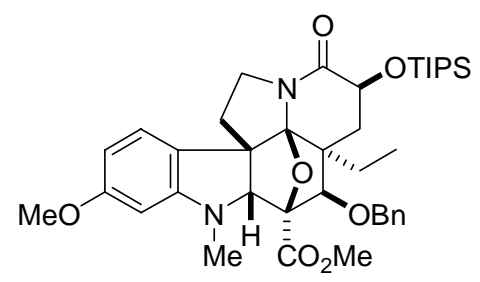

A stirred solution of $\mathbf{5 a}(27.3 \mathrm{mg}, 0.053 \mathrm{mmol})$ in anhydrous THF $(1 \mathrm{~mL})$ under Ar at $-40{ }^{\circ} \mathrm{C}$ was treated with bis(trimethylsilyl)peroxide $(0.042 \mathrm{~mL}, 0.212 \mathrm{mmol})$, followed by a 1.65 $M$ solution of lithium diisopropylamide (LDA, $0.064 \mathrm{~mL}$ in THF, $0.106 \mathrm{mmol}$ ). The reaction 
mixture was stirred for $1 \mathrm{~h}$ at $-40{ }^{\circ} \mathrm{C}$, followed by another three additions of bis(trimethylsilyl)peroxide $(0.042 \mathrm{~mL}, 0.212 \mathrm{mmol})$ and LDA $(0.064 \mathrm{~mL}, 0.106 \mathrm{mmol})$ every $1 \mathrm{~h}$. The reaction mixture was treated with triisopropylsilyl trifluoromethanesulfonate $(0.110 \mathrm{~mL}$, $0.415 \mathrm{mmol}$ ) at $-40{ }^{\circ} \mathrm{C}$, and the mixture was allowed to warm to room temperature over $2 \mathrm{~h}$. The mixture was diluted with saturated aqueous $\mathrm{NH}_{4} \mathrm{Cl}$ and was extracted with EtOAc $(3 \times 5$ $\mathrm{mL}$ ). The extract was washed with saturated aqueous $\mathrm{NaCl}$, dried over $\mathrm{MgSO}_{4}$, and concentrated under reduced pressure. Flash chromatography $\left(\mathrm{SiO}_{2}, 10 \%\right.$ EtOAc-hexanes) gave $23.3 \mathrm{mg}(64 \%)$ of 12 as a white foam: natural enantiomer $[\alpha]_{D}^{23}+53(c 1.6, \mathrm{MeOH})$, unnatural enantiomer $[\alpha]_{\mathrm{D}}{ }^{23}-50(c 1.15, \mathrm{MeOH}) ;{ }^{1} \mathrm{H}$ NMR $\left(500 \mathrm{MHz}, \mathrm{C}_{6} \mathrm{D}_{6}\right) \delta 7.26(\mathrm{~d}, J=7.3 \mathrm{~Hz}, 2 \mathrm{H})$, $7.07-7.18(\mathrm{~m}, 3 \mathrm{H}), 6.42(\mathrm{~d}, J=8.1 \mathrm{~Hz}, 1 \mathrm{H}), 6.16(\mathrm{dd}, J=2.2,8.1 \mathrm{~Hz}, 1 \mathrm{H}), 6.08(\mathrm{~d}, J=2.2 \mathrm{~Hz}$, $1 \mathrm{H}), 4.57(\mathrm{~d}, J=11.4 \mathrm{~Hz}, 1 \mathrm{H}), 4.53(\mathrm{dd}, J=5.5,11.7 \mathrm{~Hz}, 1 \mathrm{H}), 4.36(\mathrm{~d}, J=11.4 \mathrm{~Hz}, 1 \mathrm{H}), 4.26$ $(\mathrm{s}, 1 \mathrm{H}), 3.82(\mathrm{dd}, J=10.6,11.0 \mathrm{~Hz}, 1 \mathrm{H}), 3.72-3.78(\mathrm{~m}, 1 \mathrm{H}), 3.74(\mathrm{~s}, 1 \mathrm{H}), 3.37(\mathrm{~s}, 3 \mathrm{H}), 3.32$ $(\mathrm{s}, 3 \mathrm{H}), 3.08(\mathrm{t}, J=12.8 \mathrm{~Hz}, 1 \mathrm{H}), 2.83(\mathrm{~s}, 3 \mathrm{H}), 2.35(\mathrm{dd}, J=5.5,13.9 \mathrm{~Hz}, 1 \mathrm{H}), 2.08(\mathrm{dt}, J=$ $10.3,12.5 \mathrm{~Hz}, 1 \mathrm{H}), 1.63(\mathrm{~m}, 1 \mathrm{H}), 1.05-1.35(\mathrm{~m}, 23 \mathrm{H}), 0.70(\mathrm{t}, J=7.4 \mathrm{~Hz}, 3 \mathrm{H}) ;{ }^{13} \mathrm{C}$ NMR $(125$ $\left.\mathrm{MHz}, \mathrm{C}_{6} \mathrm{D}_{6}\right) \delta 170.7,170.4,162.3,155.1,138.7,129.0(2 \mathrm{C}), 128.1(2 \mathrm{C}), 124.4,121.9,104.8$, 103.7, 95.1, 91.6, 88.1, 82.3, 74.8, 68.5, 64.3, 55.1, 51.9, 51.0, 47.1, 38.3, 35.5, 32.7, 30.4, 24.2, 18.7 (6C), 13.1 (3C), 10.5; IR (neat) 2940, 2865, 1732, 1683, 1620, $1500 \mathrm{~cm}^{-1}$; HRMS (MALDI-FTMS) calcd for $\left(\mathrm{C}_{39} \mathrm{H}_{55} \mathrm{~N}_{2} \mathrm{O}_{7} \mathrm{Si}\right)[\mathrm{M}+\mathrm{H}]^{+}$691.3773; found 691.3787 .

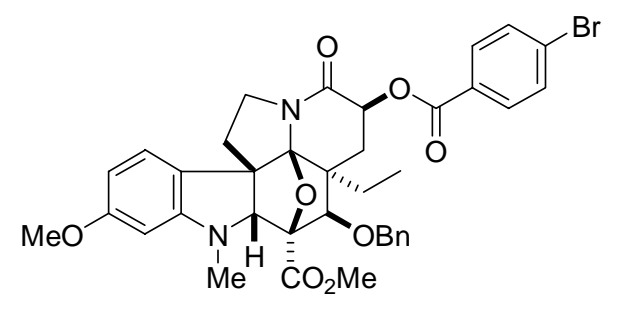

A solution of $12(10.0 \mathrm{mg}, 0.015 \mathrm{mmol})$ in THF $(3 \mathrm{~mL})$ was treated with a $1.0 \mathrm{M}$ solution of $\mathrm{Bu}_{4} \mathrm{NF}(0.025 \mathrm{~mL}, 0.025 \mathrm{mmol})$ at room temperature, and the mixture was stirred for $30 \mathrm{~min}$. The reaction mixture was concentrated under reduced pressure to give a yellow oil. A solution of $p$-bromobenzoyl chloride $(10 \mathrm{mg}, 0.05 \mathrm{mmol})$ in $\mathrm{CH}_{2} \mathrm{Cl}_{2}(3 \mathrm{~mL})$ was treated with $\mathrm{Et}_{3} \mathrm{~N}(15 \mathrm{mg}$, $0.14 \mathrm{mmol}), \operatorname{DMAP}(1.7 \mathrm{mg}, 0.015 \mathrm{mmol})$ and a solution of the above mixture in $\mathrm{CH}_{2} \mathrm{Cl}_{2}(1 \mathrm{~mL})$, and the reaction mixture was stirred at room temperature for $1 \mathrm{~h}$. The reaction mixture was concentrated under reduced pressure. Flash chromatography $\left(\mathrm{SiO}_{2}, 25 \%\right.$ EtOAc-hexanes) gave $8.7 \mathrm{mg}$ (81\%, two steps) of $\mathbf{1 2 a}$ as a white solid. A sample of $\mathbf{1 2 a}$ (CCDC 255976) for X-ray analysis was obtained as block shaped crystals by recrystallization from EtOAc-hexanes: $\mathrm{mp}$ 
223-224 ${ }^{\circ} \mathrm{C} ;{ }^{1} \mathrm{H}$ NMR $\left(500 \mathrm{MHz}, \mathrm{C}_{6} \mathrm{D}_{6}\right) \delta 7.67(\mathrm{~d}, J=8.4 \mathrm{~Hz}, 2 \mathrm{H}), 7.11-7.17(\mathrm{~m}, 2 \mathrm{H}), 7.07(\mathrm{~d}, J$ $=8.4 \mathrm{~Hz}, 2 \mathrm{H}), 7.02-7.05(\mathrm{~m}, 2 \mathrm{H}), 6.97-7.01(\mathrm{~m}, 1 \mathrm{H}), 6.38(\mathrm{~d}, J=8.1 \mathrm{~Hz}, 1 \mathrm{H}), 6.21(\mathrm{dd}, J=$ $2.2,8.1 \mathrm{~Hz}, 1 \mathrm{H}), 6.07(\mathrm{~d}, J=1.8 \mathrm{~Hz}, 1 \mathrm{H}), 5.97(\mathrm{dd}, J=5.9,12.8 \mathrm{~Hz}, 1 \mathrm{H}), 4.44(\mathrm{~d}, J=11.0 \mathrm{~Hz}$, $1 \mathrm{H}), 4.30(\mathrm{~s}, 1 \mathrm{H}), 4.29(\mathrm{~d}, J=11.0 \mathrm{~Hz}, 1 \mathrm{H}), 3.94(\mathrm{t}, J=11.0 \mathrm{~Hz}, 1 \mathrm{H}), 3.77(\mathrm{~s}, 1 \mathrm{H}), 3.68-3.75$ $(\mathrm{m}, 1 \mathrm{H}), 3.39(\mathrm{~s}, 6 \mathrm{H}), 3.16(\mathrm{t}, J=13.1 \mathrm{~Hz}, 1 \mathrm{H}), 2.81(\mathrm{~s}, 3 \mathrm{H}), 2.30(\mathrm{dd}, J=5.9,13.6 \mathrm{~Hz}, 1 \mathrm{H})$, $2.08(\mathrm{dt}, J=10.5,12.5 \mathrm{~Hz}, 1 \mathrm{H}), 1.68(\mathrm{dd}, J=7.9,12.5 \mathrm{~Hz}, 1 \mathrm{H}), 0.85-1.02(\mathrm{~m}, 2 \mathrm{H}), 0.61-0.69$ $(\mathrm{m}, 3 \mathrm{H}) ;{ }^{13} \mathrm{C}$ NMR $\left(125 \mathrm{MHz}, \mathrm{C}_{6} \mathrm{D}_{6}\right) \delta 170.3,166.9,165.5,162.4,155.0,138.3,132.1(2 \mathrm{C})$, 132.0 (2C), 129.4, 129.3, 128.9 (2C), 128.7 (2C), 124.7, 121.4, 104.7, 103.9, 95.1, 91.8, 87.9, 82.4, 75.0, 68.4, 64.4, 55.1, 51.9, 51.3, 47.1, 38.4, 35.6, 30.4, 28.5, 24.2, 10.3; HRMS (MALDI-FTMS) calcd for $\left(\mathrm{C}_{37} \mathrm{H}_{38} \mathrm{BrN}_{2} \mathrm{O}_{8}\right)[\mathrm{M}+\mathrm{H}]^{+}$717.1733; found 717.1733 .

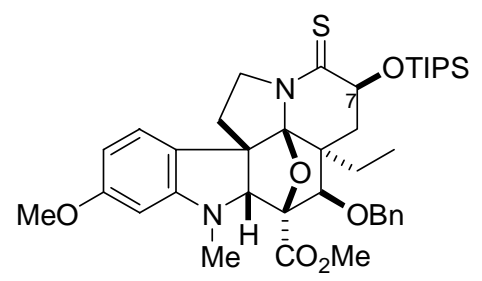

A solution of $12(52 \mathrm{mg}, 0.075 \mathrm{mmol})$ in toluene $(4 \mathrm{~mL})$ was treated with Lawesson's reagent $(61 \mathrm{mg}, 0.151 \mathrm{mmol}$ ) at room temperature, and the heterogeneous mixture was warmed at $110{ }^{\circ} \mathrm{C}$ for $3 \mathrm{~h}$. Upon completion, the now homogeneous yellow solution was cooled to room temperature, and concentrated under reduced pressure. Flash chromatography $\left(\mathrm{SiO}_{2}\right.$, 25\% EtOAc-hexanes) followed by PTLC (25\% EtOAc-hexanes) gave $\mathbf{1 3}(37.3 \mathrm{mg}, 70 \%)$ and its C7 epimer $(5.3 \mathrm{mg}, 10 \%)$ as yellow solids.

For 13: natural enantiomer $[\alpha]_{\mathrm{D}}^{23}+96\left(c 0.68, \mathrm{CHCl}_{3}\right)$, unnatural enantiomer $[\alpha]_{\mathrm{D}}^{23}-94$ (c 2.8, $\left.\mathrm{CHCl}_{3}\right) ;{ }^{1} \mathrm{H}$ NMR $\left(500 \mathrm{MHz}, \mathrm{C}_{6} \mathrm{D}_{6}\right) \delta 7.28(\mathrm{~d}, J=7.3 \mathrm{~Hz}, 2 \mathrm{H}), 7.07-7.21(\mathrm{~m}, 3 \mathrm{H}), 6.48(\mathrm{~d}$, $J=8.1 \mathrm{~Hz}, 1 \mathrm{H}), 6.12(\mathrm{dd}, J=2.2,8.1 \mathrm{~Hz}, 1 \mathrm{H}), 6.09(\mathrm{~d}, J=2.2 \mathrm{~Hz}, 1 \mathrm{H}), 4.73(\mathrm{dd}, J=5.5,11.4$ $\mathrm{Hz}, 1 \mathrm{H}), 4.58(\mathrm{~d}, J=11.5 \mathrm{~Hz}, 1 \mathrm{H}), 4.35(\mathrm{~d}, J=11.0 \mathrm{~Hz}, 1 \mathrm{H}), 4.31(\mathrm{~s}, 1 \mathrm{H}), 4.21$ (ddd, $J=8.1$, $10.6,13.9 \mathrm{~Hz}, 1 \mathrm{H}), 4.13(\mathrm{dd}, J=9.5,13.9 \mathrm{~Hz}, 1 \mathrm{H}), 3.73(\mathrm{~s}, 1 \mathrm{H}), 3.37(\mathrm{~s}, 3 \mathrm{H}), 3.31(\mathrm{~s}, 3 \mathrm{H})$, $2.99(\mathrm{dd}, J=12.8,12.8 \mathrm{~Hz}, 1 \mathrm{H}), 2.83(\mathrm{~s}, 3 \mathrm{H}), 2.38(\mathrm{dd}, J=5.5,13.9 \mathrm{~Hz}, 1 \mathrm{H}), 2.10(\mathrm{dt}, J=$ 10.6, $12.5 \mathrm{~Hz}, 1 \mathrm{H}), 1.68(\mathrm{dd}, J=7.0,12.5 \mathrm{~Hz}, 1 \mathrm{H}), 1.20-1.37(\mathrm{~m}, 21 \mathrm{H}), 1.03(\mathrm{dq}, J=7.3,14.7$ $\mathrm{Hz}, 1 \mathrm{H}), 0.78(\mathrm{dq}, J=7.3,14.7 \mathrm{~Hz}, 1 \mathrm{H}), 0.63(\mathrm{t}, J=7.4 \mathrm{~Hz}, 3 \mathrm{H}) ;{ }^{13} \mathrm{C} \mathrm{NMR}\left(125 \mathrm{MHz}, \mathrm{C}_{6} \mathrm{D}_{6}\right)$ $\delta$ 204.2, 170.1, 162.2, 154.9, 138.3, 129.0 (2C), 128.5, 128.1 (2C), 124.1, 121.1, 103.5, 102.0, $95.0,91.7,87.5,81.9,74.5,73.6,64.7,54.8,54.5,51.6,51.0,37.6,35.2,32.3,23.8,18.7$ 
(3C), 18.6 (3C), 13.2 (3C), 10.2; IR (neat) 2942, 2865, 1765, 1732, 1621, 1591, 1500, 1422, 1356, 1246, 1202, $1102 \mathrm{~cm}^{-1}$; HRMS (MALDI-FTMS) calcd for $\left(\mathrm{C}_{39} \mathrm{H}_{55} \mathrm{~N}_{2} \mathrm{O}_{6} \mathrm{SSi}\right)[\mathrm{M}+\mathrm{H}]^{+}$ 707.3544; found 707.3519.

For the $\mathrm{C} 7$ epimer of 13: natural enantiomer $[\alpha]_{\mathrm{D}}{ }^{23}+106\left(c 0.28, \mathrm{CHCl}_{3}\right)$, unnatural enantiomer $[\alpha]_{\mathrm{D}}{ }^{23}-114\left(c 0.42, \mathrm{CHCl}_{3}\right) ;{ }^{1} \mathrm{H}$ NMR $\left(500 \mathrm{MHz}, \mathrm{C}_{6} \mathrm{D}_{6}\right) \delta 7.28(\mathrm{~d}, J=7.4 \mathrm{~Hz}, 2 \mathrm{H})$, 7.10-7.21 (m, 3H), $6.62(\mathrm{~d}, J=7.7 \mathrm{~Hz}, 1 \mathrm{H}), 6.14(\mathrm{~s}, 1 \mathrm{H}), 6.12(\mathrm{dd}, J=2.2,7.7 \mathrm{~Hz}, 1 \mathrm{H}), 4.91$ $(\mathrm{dd}, J=2.9,6.2 \mathrm{~Hz}, 1 \mathrm{H}), 4.55(\mathrm{~d}, J=11.0 \mathrm{~Hz}, 1 \mathrm{H}), 4.39(\mathrm{~d}, J=11.0 \mathrm{~Hz}, 1 \mathrm{H}), 4.37(\mathrm{~s}, 1 \mathrm{H})$, $4.30-4.40(\mathrm{~m}, 1 \mathrm{H}), 4.23$ (ddd, J= 7.7, 11.0, $13.6 \mathrm{~Hz}, 1 \mathrm{H}), 3.73(\mathrm{~s}, 1 \mathrm{H}), 3.40(\mathrm{~s}, 3 \mathrm{H}), 3.33(\mathrm{~s}$, $3 \mathrm{H}), 2.95(\mathrm{dd}, J=6.4,14.9 \mathrm{~Hz}, 1 \mathrm{H}), 2.83(\mathrm{~s}, 3 \mathrm{H}), 2.08-2.17(\mathrm{~m}, 2 \mathrm{H}), 1.72(\mathrm{dd}, J=7.3,12.5$ $\mathrm{Hz}, 1 \mathrm{H}), 1.51(\mathrm{dq}, J=6.6,13.7 \mathrm{~Hz}, 1 \mathrm{H}), 1.12-1.41(\mathrm{~m}, 21 \mathrm{H}), 0.91-0.96(\mathrm{~m}, 1 \mathrm{H}), 0.71(\mathrm{t}, J=$ $7.4 \mathrm{~Hz}, 3 \mathrm{H}) ;{ }^{13} \mathrm{C}$ NMR $\left(125 \mathrm{MHz}, \mathrm{C}_{6} \mathrm{D}_{6}\right) \delta 203.4,169.9,162.1,155.0,138.3,128.4(2 \mathrm{C}), 127.6$, 127.0 (2C), 124.1, 121.3, 103.4, 102.5, 95.2, 91.4, 87.8, 82.1, 74.3, 74.2, 65.4, 54.9, 54.8, 51.6, 50.6, 37.7, 35.4, 31.2, 25.2, 18.7 (6C), 13.5 (3C), 11.0; IR (neat) 2941, 2864, 1762, 1734, 1618, 1499, 1437, 1362, 1244, $1087 \mathrm{~cm}^{-1}$; HRMS (MALDI-FTMS) calcd for $\left(\mathrm{C}_{39} \mathrm{H}_{55} \mathrm{~N}_{2} \mathrm{O}_{6} \mathrm{SSi}\right)[\mathrm{M}+\mathrm{H}]^{+}$707.3544; found 707.3539 .

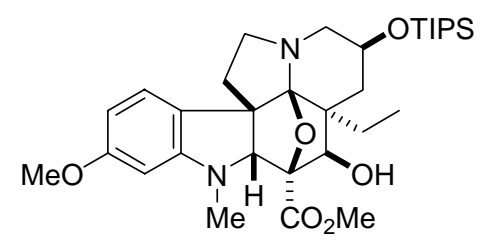

A solution of excess Raney-Ni (pretreated with successive washes with $\mathrm{H}_{2} \mathrm{O}(5 \mathrm{x})$, and THF (5x)) in THF (3 mL) was treated with a solution of $13(11 \mathrm{mg}, 0.016 \mathrm{mmol})$ in THF (1 mL), and the mixture was stirred for $15 \mathrm{~h}$ at room temperature under an atmosphere of $\mathrm{H}_{2}$. The mixture was filtered through a pad of Celite, and the filtrate was concentrated under reduced pressure to give $8.3 \mathrm{mg}(91 \%)$ of $\mathbf{1 4}$ as a yellow oil: natural enantiomer $[\alpha]_{\mathrm{D}}{ }^{23}+73(c 1.5$, $\left.\mathrm{CHCl}_{3}\right)$, unnatural enantiomer $[\alpha]_{\mathrm{D}}{ }^{23}-72\left(c 0.52, \mathrm{CHCl}_{3}\right) ;{ }^{1} \mathrm{H}$ NMR $\left(500 \mathrm{MHz}, \mathrm{C}_{6} \mathrm{D}_{6}\right) \delta 6.90(\mathrm{~d}, J$ $=8.1 \mathrm{~Hz}, 1 \mathrm{H}), 6.30(\mathrm{dd}, J=2.2,8.1 \mathrm{~Hz}, 1 \mathrm{H}), 6.16(\mathrm{~d}, J=2.2 \mathrm{~Hz}, 1 \mathrm{H}), 4.50(\mathrm{~d}, J=7.5 \mathrm{~Hz}$, $1 \mathrm{H}), 4.28-4.32(\mathrm{~m}, 1 \mathrm{H}), 3.92(\mathrm{~s}, 1 \mathrm{H}), 3.40(\mathrm{~s}, 3 \mathrm{H}), 3.38(\mathrm{~s}, 3 \mathrm{H}), 3.17-3.25(\mathrm{~m}, 2 \mathrm{H}), 2.95-3.04$ $(\mathrm{m}, 2 \mathrm{H}), 2.81(\mathrm{~s}, 3 \mathrm{H}), 2.35(\mathrm{dd}, J=12.3,12.3 \mathrm{~Hz}, 1 \mathrm{H}), 2.10-2.18(\mathrm{~m}, 2 \mathrm{H}), 1.96-2.05(\mathrm{~m}, 1 \mathrm{H})$, $1.90-1.94(\mathrm{~m}, 1 \mathrm{H}), 1.21-1.38(\mathrm{~m}, 2 \mathrm{H}), 0.96-1.06(\mathrm{~m}, 21 \mathrm{H}), 0.82(\mathrm{t}, J=7.3 \mathrm{~Hz}, 3 \mathrm{H}) ;{ }^{13} \mathrm{C} \mathrm{NMR}$ $\left(125 \mathrm{MHz}, \mathrm{C}_{6} \mathrm{D}_{6}\right) \delta 171.2,161.9,154.5,128.2,125.1,124.5,102.9,94.4,89.5,84.5,79.7,66.2$, $62.5,55.1,54.8,51.4,51.349 .4,41.2,35.3,32.8,24.9,18.4$ (6C), 12.6 (3C), 10.1; IR (neat) 
$3498,2938,2856,1729,1609,1506,1470,1345,1268,1247 \mathrm{~cm}^{-1}$; HRMS (MALDI-FTMS) calcd for $\left(\mathrm{C}_{32} \mathrm{H}_{51} \mathrm{~N}_{2} \mathrm{O}_{6} \mathrm{Si}\right)[\mathrm{M}+\mathrm{H}]^{+} 587.3511$ found 587.3504 .

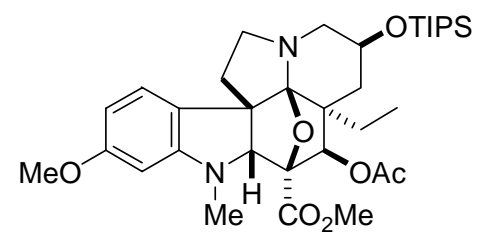

A mixture of $14(9.0 \mathrm{mg}, 0.015 \mathrm{mmol})$ and $\mathrm{NaOAc}(6.3 \mathrm{mg}, 0.075 \mathrm{mmol})$ was treated with acetic anhydride $(0.5 \mathrm{~mL})$, and the mixture was stirred for $17 \mathrm{~h}$ at room temperature. The reaction mixture was diluted with $\mathrm{CH}_{2} \mathrm{Cl}_{2}(2 \mathrm{~mL})$ and washed with saturated aqueous $\mathrm{NaHCO}_{3}$. The aqueous phase was extracted with $\mathrm{CH}_{2} \mathrm{Cl}_{2}(3 \times 3 \mathrm{~mL})$. The combined organic mixture was washed with saturated aqueous $\mathrm{NaCl}$, dried over $\mathrm{MgSO}_{4}$, and concentrated under reduced pressure. Flash chromatography $\left(\mathrm{SiO}_{2}, 50 \%\right.$ EtOAc-hexanes) provided $9.1 \mathrm{mg}(97 \%)$ of $\mathbf{1 5}$ as a yellow oil: natural enantiomer $[\alpha]_{\mathrm{D}}^{23}+41\left(c 1.2, \mathrm{CHCl}_{3}\right),[\alpha]_{\mathrm{D}}^{25}+50\left(c 0.36, \mathrm{CHCl}_{3}\right)$, unnatural enantiomer $[\alpha]_{\mathrm{D}}{ }^{23}-40\left(c 1.3, \mathrm{CHCl}_{3}\right) ;{ }^{1} \mathrm{H}$ NMR $\left(500 \mathrm{MHz}, \mathrm{C}_{6} \mathrm{D}_{6}\right) \delta 6.89(\mathrm{~d}, J=8.1 \mathrm{~Hz}, 2 \mathrm{H}), 6.33$ (dd, $J=2.2,8.1 \mathrm{~Hz}, 1 \mathrm{H}), 6.07(\mathrm{~d}, J=2.2 \mathrm{~Hz}, 1 \mathrm{H}), 6.07(\mathrm{~s}, 1 \mathrm{H}), 4.21-4.32(\mathrm{~m}, 1 \mathrm{H}), 3.99(\mathrm{~s}$, $1 \mathrm{H}), 3.40(\mathrm{~s}, 6 \mathrm{H}), 3.19-3.27(\mathrm{~m}, 2 \mathrm{H}), 3.01(\mathrm{t}, J=9.7 \mathrm{~Hz}, 1 \mathrm{H}), 2.97-3.01(\mathrm{~m}, 1 \mathrm{H}), 2.90(\mathrm{~s}, 3 \mathrm{H})$, 2.50 (dd, $J=12.3,12.3 \mathrm{~Hz}, 1 \mathrm{H}), 2.18(\mathrm{ddd}, J=4.4,10.6,13.0 \mathrm{~Hz}, 1 \mathrm{H}), 2.04-2.10(\mathrm{~m}, 1 \mathrm{H})$, $2.02(\mathrm{dd}, J=2.9,13.2 \mathrm{~Hz}, 1 \mathrm{H}), 1.63(\mathrm{~s}, 3 \mathrm{H}), 1.31-1.45(\mathrm{~m}, 1 \mathrm{H}), 1.00-1.15(\mathrm{~m}, 22 \mathrm{H}), 0.85(\mathrm{t}, J$ $=7.3 \mathrm{~Hz}, 3 \mathrm{H}) ;{ }^{13} \mathrm{C}$ NMR $\left(125 \mathrm{MHz}, \mathrm{C}_{6} \mathrm{D}_{6}\right) \delta 170.3,169.3,162.5,154.8,125.3,124.1,110.2$, 103.7, 94.5, 89.0, 84.7, 80.4, 66.4, 63.0, 55.4, 55.2, 51.7, 49.9, 45.8, 41.0, 35.2, 34.3, 25.2, 20.6, 18.7 (6C), 13.1 (3C), 10.6; IR (neat) 2944, 2866, 1753,1731,1612, $1501 \mathrm{~cm}^{-1}$; HRMS (MALDI-FTMS) calcd for $\left(\mathrm{C}_{34} \mathrm{H}_{53} \mathrm{~N}_{2} \mathrm{O}_{7} \mathrm{Si}\right)[\mathrm{M}+\mathrm{H}]^{+}$629.3616; found 629.3605 .

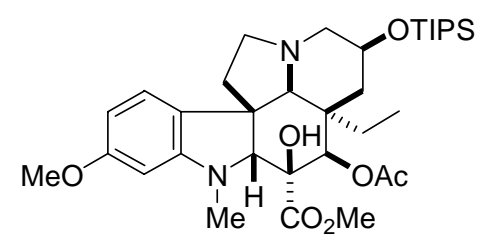

A solution of $15(25 \mathrm{mg}, 0.040 \mathrm{mmol})$ in $\mathrm{MeOH}(2.5 \mathrm{~mL})$ and EtOAc $(2.5 \mathrm{~mL})$ was sonicated to give a homogeneous solution. This mixture was treated with $\mathrm{PtO}_{2}(5.0 \mathrm{mg})$ in one portion, and the mixture was stirred under $40-45$ psi of $\mathrm{H}_{2}$ for $3 \mathrm{~h}$. The resulting mixture was filtered through a pad of Celite (EtOAc eluent) to remove the catalyst. The filtrate was concentrated under reduced pressure to give $24.6 \mathrm{mg}(98 \%)$ of $\mathbf{1 6}$ as a yellow oil: natural 
enantiomer $[\alpha]_{D}{ }^{23}-28\left(c 1.15, \mathrm{CHCl}_{3}\right)$, unnatural enantiomer $[\alpha]_{\mathrm{D}}{ }^{23}+32\left(\mathrm{c} \quad 0.66, \mathrm{CHCl}_{3}\right) ;{ }^{1} \mathrm{H}$ NMR (500 MHz, $\left.\mathrm{C}_{6} \mathrm{D}_{6}\right) \delta 8.05-8.09(\mathrm{~m}, 1 \mathrm{H}), 6.61(\mathrm{~d}, J=8.2 \mathrm{~Hz}, 1 \mathrm{H}), 6.41(\mathrm{dd}, J=2.2,8.2 \mathrm{~Hz}$, $1 \mathrm{H}), 5.98(\mathrm{~d}, J=2.2 \mathrm{~Hz}, 1 \mathrm{H}), 5.91(\mathrm{~s}, 1 \mathrm{H}), 4.18-4.23(\mathrm{~m}, 1 \mathrm{H}), 3.73(\mathrm{~s}, 1 \mathrm{H}), 3.43(\mathrm{~s}, 3 \mathrm{H}), 3.39$ $(\mathrm{s}, 3 \mathrm{H}), 2.96(\mathrm{dd}, J=5.8,11.1 \mathrm{~Hz}, 1 \mathrm{H}), 2.86-2.91(\mathrm{~m}, 1 \mathrm{H}), 2.81(\mathrm{dd}, J=11.8,13.7 \mathrm{~Hz}, 1 \mathrm{H})$, $2.50(\mathrm{~s}, 3 \mathrm{H}), 2.43-2.48(\mathrm{~m}, 1 \mathrm{H}), 2.37(\mathrm{~s}, 1 \mathrm{H}), 1.91-2.02(\mathrm{~m}, 3 \mathrm{H}), 1.84(\mathrm{~s}, 3 \mathrm{H}), 1.64-1.67(\mathrm{~m}$, $1 \mathrm{H}), 1.41(\mathrm{dq}, J=7.5,14.9 \mathrm{~Hz}, 1 \mathrm{H}), 1.05-1.09(\mathrm{~m}, 21 \mathrm{H}), 1.03-1.05(\mathrm{~m}, 1 \mathrm{H}), 0.65(\mathrm{t}, J=7.5$ $\mathrm{Hz}, 3 \mathrm{H}) ;{ }^{13} \mathrm{C}$ NMR $\left(125 \mathrm{MHz}, \mathrm{C}_{6} \mathrm{D}_{6}\right) \delta 171.7,169.8,161.7,154.3,125.8,123.0,105.4,95.9$, 83.9, 80.4, 73.7, 71.3, 65.8, 60.9, 54.8, 52.9, 52.7, 51.2, 46.2, 40.6, 38.4, 38.3, 33.8, 20.8, $18.2(6 \mathrm{C}), 12.6(3 \mathrm{C}), 8.6$; HRMS (MALDI-FTMS) calcd for $\left(\mathrm{C}_{34} \mathrm{H}_{54} \mathrm{~N}_{2} \mathrm{O}_{7} \mathrm{Si}\right)[\mathrm{M}+\mathrm{H}]^{+} 631.3773$; found 631.3777 .

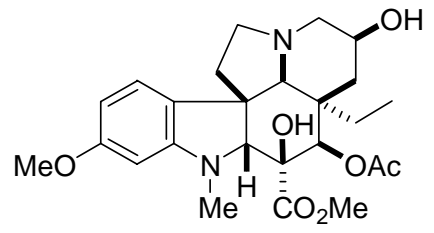

A solution of $16(16.0 \mathrm{mg}, 0.025 \mathrm{mmol})$ in THF $(2 \mathrm{~mL})$ was treated with a $1.0 \mathrm{M}$ solution of $\mathrm{Bu}_{4} \mathrm{NF}(0.05 \mathrm{~mL}$ in $\mathrm{THF}, 0.05 \mathrm{mmol})$ at room temperature, and the mixture was stirred for 30 min. The reaction mixture was concentrated under reduced pressure. Flash chromatography $\left(\mathrm{SiO}_{2}, 5 \% \mathrm{Et}_{3} \mathrm{~N}-\mathrm{EtOAC}\right)$ provided $10.6 \mathrm{mg}(89 \%)$ of $\mathbf{1 7}$ as a yellow oil: natural enantiomer $[\alpha]_{\mathrm{D}}{ }^{23}+24\left(c 0.71, \mathrm{CHCl}_{3}\right)$, unnatural enantiomer $[\alpha]_{\mathrm{D}}{ }^{23}-24\left(c 0.75, \mathrm{CHCl}_{3}\right) ;{ }^{1} \mathrm{H}$ NMR $(500 \mathrm{MHz}$, $\left.\mathrm{C}_{6} \mathrm{D}_{6}\right) \delta 6.58(\mathrm{~d}, J=8.1 \mathrm{~Hz}, 1 \mathrm{H}), 6.35(\mathrm{dd}, J=2.2,8.1 \mathrm{~Hz}, 1 \mathrm{H}), 6.03(\mathrm{~d}, J=2.2 \mathrm{~Hz}, 1 \mathrm{H}), 5.98$ $(\mathrm{s}, 1 \mathrm{H}), 4.21-4.32(\mathrm{~m}, 1 \mathrm{H}), 3.99(\mathrm{~s}, 1 \mathrm{H}), 3.73-3.79(\mathrm{~m}, 1 \mathrm{H}), 3.41(\mathrm{~s}, 3 \mathrm{H}), 3.40(\mathrm{~s}, 3 \mathrm{H}), 2.99(\mathrm{~d}$, $J=11.7 \mathrm{~Hz}, 1 \mathrm{H}), 2.67(\mathrm{t}, J=8.6 \mathrm{~Hz}, 1 \mathrm{H}), 2.35(\mathrm{~s}, 3 \mathrm{H}), 2.20$ (ddd, $J=9.2,10.3,13.6 \mathrm{~Hz}, 1 \mathrm{H}$ ), $1.94-2.00(\mathrm{~m}, 1 \mathrm{H}), 1.89(\mathrm{~s}, 3 \mathrm{H}), 1.76-1.88(\mathrm{~m}, 3 \mathrm{H}), 1.68(\mathrm{~d}, J=16.1 \mathrm{~Hz}, 1 \mathrm{H}), 1.61(\mathrm{dd}, J=$ $2.2,11.4 \mathrm{~Hz}, 1 \mathrm{H}), 1.29-1.38(\mathrm{~m}, 1 \mathrm{H}), 1.25(\mathrm{dq}, J=7.2,14.7 \mathrm{~Hz}, 1 \mathrm{H}), 1.14(\mathrm{dd}, J=4.2,15.4$ $\mathrm{Hz}, 1 \mathrm{H}), 0.49(\mathrm{t}, J=7.3 \mathrm{~Hz}, 3 \mathrm{H}) ;{ }^{13} \mathrm{C}$ NMR $\left(125 \mathrm{MHz}, \mathrm{C}_{6} \mathrm{D}_{6}\right) \delta 172.8,169.4,162.1,155.0,127.4$, 123.7, 105.6, 96.7, 84.0, 79.8, 76.5, 73.6, 65.1, 59.8, 55.3, 53.7, 53.2, 51.9, 44.1, 41.1, 37.9, 35.8, 34.6, 21.1, 8.5; IR (neat) 3549, 2952, 1746, 1615, 1504, 1435, $1225 \mathrm{~cm}^{-1}$; HRMS (MALDI-FTMS) calcd for $\left(\mathrm{C}_{25} \mathrm{H}_{35} \mathrm{~N}_{2} \mathrm{O}_{7}\right)[\mathrm{M}+\mathrm{H}]^{+} 475.2439$; found 475.2443 . 


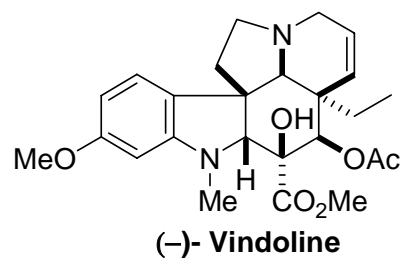

Vindoline (3). DEAD (4.7 $\mu \mathrm{L}, 0.029 \mathrm{mmol})$ was added to a solution of $17(4.6 \mathrm{mg}$, $0.0097 \mathrm{mmol})$ and $\mathrm{Ph}_{3} \mathrm{P}(7.7 \mathrm{mg}, 0.029 \mathrm{mmol})$ in anhydrous THF $(100 \mu \mathrm{L})$ at room temperature under Ar. The reaction mixture was stirred for $24 \mathrm{~h}$ at room temperature in the dark, before the solvent was removed under reduced pressure. PTLC $\left(\mathrm{SiO}_{2}, 75 \%\right.$ EtOAc-hexanes) afforded vindoline (3) as a white amorphous solid (3.3 $\mathrm{mg}, 75 \%)$ identical in all respects with authentic material. For synthetic natural vindoline (3): $\left.[\alpha]_{D}^{20}-45(c 0.14, \mathrm{MeOH})\right),-17\left(c 0.40, \mathrm{CHCl}_{3}\right)$; for ent-vindoline (3): $[\alpha]_{\mathrm{D}}{ }^{25}+46(c 0.19, \mathrm{MeOH})$; for authentic natural vindoline: $[\alpha]_{\mathrm{D}}^{20}-48(c$ $0.19, \mathrm{MeOH}),-19\left(\right.$ c 0.30, $\left.\mathrm{CHCl}_{3}\right), \mathrm{lit}^{55}[\alpha]_{\mathrm{D}}{ }^{20}-18\left(c 0.20, \mathrm{CHCl}_{3}\right) ;{ }^{1} \mathrm{H}$ NMR $\left(500 \mathrm{MHz}, \mathrm{C}_{6} \mathrm{D}_{6}\right) \delta$ $8.52(\mathrm{~s}, 1 \mathrm{H}), 6.65(\mathrm{~d}, J=8.1 \mathrm{~Hz}, 1 \mathrm{H}), 6.37(\mathrm{dd}, J=2.2,8.1 \mathrm{~Hz}, 1 \mathrm{H}), 6.05(\mathrm{~d}, J=2.2 \mathrm{~Hz}, 1 \mathrm{H})$, $5.97(\mathrm{~s}, 1 \mathrm{H}$ ), 5.47 (ddd, $J=1.4,4.7,10.2 \mathrm{~Hz}, 1 \mathrm{H}), 5.27(\mathrm{~d}, J=10.2 \mathrm{~Hz}, 1 \mathrm{H}), 3.83(\mathrm{~s}, 1 \mathrm{H}), 3.40$ $(\mathrm{s}, 3 \mathrm{H}), 3.39(\mathrm{~s}, 3 \mathrm{H}), 2.88-2.94(\mathrm{~m}, 1 \mathrm{H}), 2.86(\mathrm{dt}, J=2.2,9.2 \mathrm{~Hz}, 1 \mathrm{H}), 2.41(\mathrm{~s}, 3 \mathrm{H}), 2.20-2.28$ $(\mathrm{m}, 2 \mathrm{H}), 2.13(\mathrm{dq}, J=7.3,14.7 \mathrm{~Hz}, 1 \mathrm{H}), 2.02(\mathrm{ddd}, J=2.2,9.5,13.5 \mathrm{~Hz}, 1 \mathrm{H}), 1.90(\mathrm{~s}, 3 \mathrm{H})$, $1.85-1.89(\mathrm{~m}, 1 \mathrm{H}), 1.55(\mathrm{dq}, J=7.3,14.7 \mathrm{~Hz}, 1 \mathrm{H}), 0.47(\mathrm{t}, J=7.3 \mathrm{~Hz}, 3 \mathrm{H}) ;{ }^{13} \mathrm{C}$ NMR $(150$ $\mathrm{MHz}, \mathrm{C}_{6} \mathrm{D}_{6}$ ) $\delta 172.5,170.5,131.3,125.0-128.0$ (3C, overlapped $\mathrm{C}_{6} \mathrm{D}_{6}$ ), 124.1, 123.1, 105.2, 96.2, 83.7, 79.4, 76.6, 68.7, 54.9, 53.2, 52.6, 51.6, 51.5, 44.0, 43.8, 37.6, 31.6, 20.9, 8.3; IR (neat) 2962,1740,1616,1501,1371,1242,1032, $735 \mathrm{~cm}^{-1}$; HRMS (MALDI-FTMS) calcd for $\left(\mathrm{C}_{25} \mathrm{H}_{33} \mathrm{~N}_{2} \mathrm{O}_{6}\right)[\mathrm{M}+\mathrm{H}]^{+}$457.2333; found 457.2337.

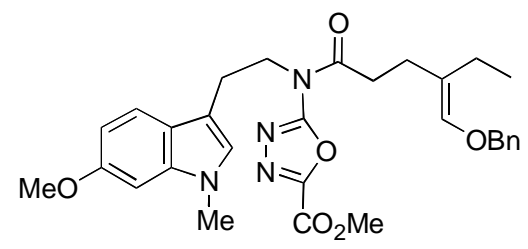

Oxadiazole $10(330 \mathrm{mg}, 1.00 \mathrm{mmol})$ was added in one portion to a solution of $(E)-\mathbf{1 1}$ (584 mg, $2.50 \mathrm{mmol}$ ) in anhydrous $\mathrm{CH}_{2} \mathrm{Cl}_{2}\left(53 \mathrm{~mL}\right.$ ) at $25{ }^{\circ} \mathrm{C}$ under Ar. The mixture was cooled to $0{ }^{\circ} \mathrm{C}$ and $\mathrm{EDCI}(479 \mathrm{mg}, 2.50 \mathrm{mmol}$ ) was added. The resulting mixture was stirred for $5 \mathrm{~min}$, before the addition of DMAP (305 mg, $2.50 \mathrm{mmol}$ ). The reaction mixture was stirred at room 
temperature overnight, before the solvent was removed under reduced pressure absorbing the mixture onto Celite. Flash chromatography $\left(\mathrm{SiO}_{2}, 30 \%\right.$ EtOAc-hexanes) afforded $\mathbf{4} \mathbf{b}$ as a white solid (480 mg, 88\%): mp 69-70 ${ }^{\circ} \mathrm{C} ;{ }^{1} \mathrm{H}$ NMR $\left(600 \mathrm{MHz}, \mathrm{CDCl}_{3}\right) \delta 7.53(\mathrm{~d}, J=9.1 \mathrm{~Hz}, 1 \mathrm{H}), 7.25-$ $7.36(\mathrm{~m}, 5 \mathrm{H}), 6.78(\mathrm{dd}, J=2.2,8.8 \mathrm{~Hz}, 1 \mathrm{H}), 6.72(\mathrm{~s}, 1 \mathrm{H}), 6.68(\mathrm{~d}, J=1.7 \mathrm{~Hz}, 1 \mathrm{H}), 5.93(\mathrm{~s}$, $1 \mathrm{H}), 4.73(\mathrm{~s}, 2 \mathrm{H}), 4.18(\mathrm{t}, J=7.5 \mathrm{~Hz}, 2 \mathrm{H}), 3.99(\mathrm{~s}, 3 \mathrm{H}), 3.87(\mathrm{~s}, 3 \mathrm{H}), 3.65(\mathrm{~s}, 3 \mathrm{H}), 3.05(\mathrm{t}, J=$ $7.5 \mathrm{~Hz}, 2 \mathrm{H}), 2.84(\mathrm{t}, J=7.6 \mathrm{~Hz}, 2 \mathrm{H}), 2.26(\mathrm{t}, J=7.6 \mathrm{~Hz}, 2 \mathrm{H}), 2.09(\mathrm{q}, J=7.4 \mathrm{~Hz}, 2 \mathrm{H}), 0.95(\mathrm{t}$, $J=7.5 \mathrm{~Hz}, 3 \mathrm{H}) ;{ }^{13} \mathrm{C}$ NMR $\left(150 \mathrm{MHz}, \mathrm{CDCl}_{3}\right) \delta 172.1,162.0,156.4,154.0,153.2,141.0,137.8$, $137.7,128.4(2 \mathrm{C}), 127.7,127.3(2 \mathrm{C}), 126.5,121.7,119.6,118.7,109.9,109.0,92.7,73.5$, 55.6, 53.6, 47.7, 35.5, 32.6, 26.7, 24.4, 20.1, 12.6; IR (neat) 2961, 1748, 1703, 1624, 1564, 1493, 1441, 1409, 1333, 1257, 1228, 1154, 1069, 1035, 813, $729 \mathrm{~cm}^{-1}$; HRMS (MALDI-FTMS) calcd for $\left(\mathrm{C}_{30} \mathrm{H}_{34} \mathrm{~N}_{4} \mathrm{O}_{6}\right)[\mathrm{M}+\mathrm{H}]^{+} 547.2551$; found 547.2554 .

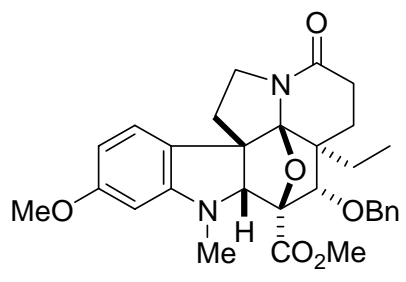

A solution of $\mathbf{4 b}(21.8 \mathrm{mg}, 0.04 \mathrm{mmol})$ in triisopropylbenzene $(40 \mathrm{~mL}, 1 \mathrm{mM})$ was purged with $\mathrm{Ar}$ for $30 \mathrm{~min}$ and was warmed at $230{ }^{\circ} \mathrm{C}$ for $20 \mathrm{~h}$. The mixture was cooled at room temperature, and loaded directly onto silica gel chromatography column. The triisopropylbenzene was eluted with hexanes, and then the column was eluted with $50 \%$ EtOAc-hexanes to afford $\mathbf{5 b}(20.4 \mathrm{mg}, 99 \%)$ as a pale yellow solid.

In earlier preparative studies, the following procedure was used. A solution of $\mathbf{4 b} \mathbf{b}(720$ $\mathrm{mg}, 1.32 \mathrm{mmol}$ ) in 1,2-dichlorobenzene ( $253 \mathrm{~mL}, 0.0052 \mathrm{M}$, passed through a column of basic alumina, degassed with Ar for $1 \mathrm{~h}$ while protected from the light) was warmed at a gentle reflux in the absence of light for $18 \mathrm{~h}$. The reaction mixture was cooled to $25{ }^{\circ} \mathrm{C}$, and loaded directly onto $\mathrm{SiO}_{2}$ equilibrated in hexanes. The 1,2-dichlorobenzene was eluted with hexanes, and then the column was eluted with 30-60\% EtOAc-hexanes (gradient eluent) to afford $\mathbf{5 b}$ (510 mg, $75 \%, 72-84 \%)$ as a white solid: $\mathrm{mp} 173-174{ }^{\circ} \mathrm{C} ;{ }^{1} \mathrm{H}$ NMR $\left(600 \mathrm{MHz}, \mathrm{C}_{6} \mathrm{D}_{6}\right) \delta 7.07-7.13(\mathrm{~m}$, $4 \mathrm{H}), 7.03-7.06(\mathrm{~m}, 1 \mathrm{H}), 6.47(\mathrm{~d}, J=8.0 \mathrm{~Hz}, 1 \mathrm{H}), 6.12(\mathrm{dd}, J=2.0,8.0 \mathrm{~Hz}, 1 \mathrm{H}), 5.83(\mathrm{~d}, J=$ $1.9 \mathrm{~Hz}, 1 \mathrm{H}), 4.66(\mathrm{~d}, J=11.7 \mathrm{~Hz}, 1 \mathrm{H}), 4.25(\mathrm{~s}, 1 \mathrm{H}), 4.17(\mathrm{~d}, J=11.7 \mathrm{~Hz}, 1 \mathrm{H}), 3.81-3.93(\mathrm{~m}$, $3 \mathrm{H}), 3.77(\mathrm{~s}, 1 \mathrm{H}), 3.37(\mathrm{~s}, 3 \mathrm{H}), 3.35(\mathrm{~s}, 3 \mathrm{H}), 2.58(\mathrm{~s}, 3 \mathrm{H}), 2.22(\mathrm{dd}, J=4.8,17.8 \mathrm{~Hz}, 1 \mathrm{H})$, 
1.99-2.17 (m, 3H), $1.76(\mathrm{dd}, J=7.6,12.3 \mathrm{~Hz}, 1 \mathrm{H}), 1.43(\mathrm{dd}, J=4.9,12.9 \mathrm{~Hz}, 1 \mathrm{H}), 1.09-1.15$ $(\mathrm{m}, 1 \mathrm{H}), 0.70-0.76(\mathrm{~m}, 1 \mathrm{H}), 0.36(\mathrm{t}, J=7.3 \mathrm{~Hz}, 3 \mathrm{H}) ;{ }^{13} \mathrm{C} \mathrm{NMR}\left(125 \mathrm{MHz}, \mathrm{CDCl}_{3}\right) \delta 171.6,170.3$, $161.7,153.9,137.9,128.1$ (2C), 127.4, 126.8, 123.7 (2C), 120.3, 107.4, 100.9, 92.3, 87.5, $85.8,81.6,74.2,63.9,55.3,52.9,46.7,46.0,38.0,33.0,29.3,29.0,18.6,9.6 ;{ }^{13} \mathrm{C}$ NMR $(150$ $\left.\mathrm{MHz}, \mathrm{C}_{6} \mathrm{D}_{6}\right) \delta 172.6,171.5,162.3,154.3,138.5,128.4(2 \mathrm{C}), 127.6(2 \mathrm{C}), 127.0,124.1,120.7$, 108.0, 101.5, 92.6, 87.8, 86.1, 82.3, 74.2, 66.3, 63.8, 54.7, 52.0, 46.9, 45.9, 38.9, 37.2, 32.9, 19.9, 9.7; IR (film) 2954, 1735, 1667, 1621, 1589, 1503, 1453, 1398, 1376, 1332, 1254, 1208, $1120,1059,1021,908,868,811,733 \mathrm{~cm}^{-1}$; HRMS (MALDI-FTMS) calcd for $\left(\mathrm{C}_{30} \mathrm{H}_{34} \mathrm{~N}_{2} \mathrm{O}_{6}\right)$ $[\mathrm{M}+\mathrm{H}]^{+}$519.2490; found 519.2482. The relative stereochemistry was confirmed upon X-ray (CCDC 253430) analysis enlisting white crystals grown from EtOAc-hexanes. The enantiomers of $\mathbf{5 b}(10 \mathrm{mg} /$ injection) were separated $(\alpha=1.19)$ on a semipreparative Chiralcel OD column $\left(2 \times 25 \mathrm{~cm}, 20 \% \mathrm{FPrOH}\right.$-hexanes, $10 \mathrm{~mL} / \mathrm{min}$ flow rate) providing $(-)-5 \mathbf{b}\left(t_{R}=27.6 \mathrm{~min}\right)$ and $(+)-5 \mathbf{b}\left(t_{R}=32.8 \mathrm{~min}\right)$. For $(-)-5 \mathbf{b}:[\alpha]_{\mathrm{D}}{ }^{23}-21(c 0.44, \mathrm{MeOH})$; for $(+)-5 \mathbf{b}:[\alpha]_{\mathrm{D}}^{23}+23(c 0.20$, $\mathrm{MeOH})$.

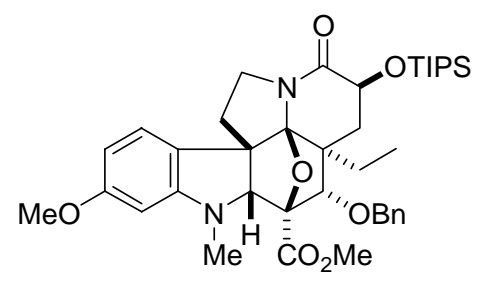

A stirred solution of $\mathbf{5 b}(28.0 \mathrm{mg}, 0.054 \mathrm{mmol})$ in anhydrous THF $(1 \mathrm{~mL})$ under Ar at $-40{ }^{\circ} \mathrm{C}$ was treated with bis(trimethylsilyl)peroxide $(0.043 \mathrm{~mL}, 0.216 \mathrm{mmol})$, followed by a 1.65 $M$ solution of lithium diisopropylamide $(0.065 \mathrm{~mL}$ in THF, $0.108 \mathrm{mmol})$. The reaction mixture was stirred for $1 \mathrm{~h}$ at $-40{ }^{\circ} \mathrm{C}$, followed by another three additions of bis(trimethylsilyl)peroxide $(0.043 \mathrm{~mL}, 0.216 \mathrm{mmol})$ and lithium diisopropylamide $(0.065 \mathrm{~mL}, 0.108 \mathrm{mmol})$ every $1 \mathrm{~h}$. The reaction mixture was treated with triisopropylsilyl trifluoromethanesulfonate $(0.118 \mathrm{~mL}, 0.440$ $\mathrm{mmol}$ ) at $-40{ }^{\circ} \mathrm{C}$, and the mixture was allowed to warm to room temperature over $2 \mathrm{~h}$. The mixture was diluted with saturated aqueous $\mathrm{NH}_{4} \mathrm{Cl}$ and was extracted with EtOAc $(3 \times 5 \mathrm{~mL})$. The extract was washed with saturated aqueous $\mathrm{NaCl}$, dried over $\mathrm{MgSO}_{4}$, and concentrated under reduced pressure. Chromatography $\left(\mathrm{SiO}_{2}, 10 \%\right.$ EtOAc-hexanes) gave $27.9 \mathrm{mg}(75 \%)$ of 18 as a white foam: ${ }^{1} \mathrm{H}$ NMR $\left(600 \mathrm{MHz}, \mathrm{C}_{6} \mathrm{D}_{6}\right) \delta 7.14-7.18(\mathrm{~m}, 4 \mathrm{H}), 7.05-7.09(\mathrm{~m}, 1 \mathrm{H}), 6.42(\mathrm{~d}$, $J=7.9 \mathrm{~Hz}, 1 \mathrm{H}), 6.13(\mathrm{dd}, J=2.2,7.9 \mathrm{~Hz}, 1 \mathrm{H}), 5.86(\mathrm{~d}, J=2.2 \mathrm{~Hz}, 1 \mathrm{H}), 4.71(\mathrm{~d}, J=11.9 \mathrm{~Hz}$, $1 \mathrm{H}), 4.53(\mathrm{dd}, J=5.7,11.9 \mathrm{~Hz}, 1 \mathrm{H}), 4.27(\mathrm{~d}, J=0.9 \mathrm{~Hz}, 1 \mathrm{H}), 4.18(\mathrm{~d}, J=11.8 \mathrm{~Hz}, 1 \mathrm{H}), 3.94$ 
$(\mathrm{d}, J=1.3 \mathrm{~Hz}, 1 \mathrm{H}), 3.74-3.81(\mathrm{~m}, 2 \mathrm{H}), 3.40(\mathrm{~s}, 3 \mathrm{H}), 3.36(\mathrm{~s}, 3 \mathrm{H}), 2.70(\mathrm{t}, J=12.3 \mathrm{~Hz}, 1 \mathrm{H})$, $2.59(\mathrm{~s}, 3 \mathrm{H}), 2.38(\mathrm{dd}, J=6.1,13.1 \mathrm{~Hz}, 1 \mathrm{H}), 2.05-2.11(\mathrm{~m}, 1 \mathrm{H}), 1.75(\mathrm{ddd}, J=2.6,7.4,12.7$ $\mathrm{Hz}, 1 \mathrm{H}), 1.29-1.38(\mathrm{~m}, 3 \mathrm{H}), 1.27(\mathrm{~d}, J=7.5 \mathrm{~Hz}, 9 \mathrm{H}), 1.23(\mathrm{~d}, J=7.5 \mathrm{~Hz}, 9 \mathrm{H}), 0.70(\mathrm{t}, J=7.4$ $\mathrm{Hz}, 3 \mathrm{H}) ;{ }^{13} \mathrm{C}$ NMR $\left(150 \mathrm{MHz}, \mathrm{C}_{6} \mathrm{D}_{6}\right) \delta 171.7,170.2,162.2,154.3,138.5,128.4$ (2C), 127.6, 127.0 (2C), 124.2, 120.9, 107.9, 101.5, 92.7, 88.0, 86.0, 82.1, 74.2, 68.2, 63.9, 54.7, 52.1, 47.0, 46.6, 40.1, 38.8, 33.0, 20.3, 18.5 (3C), 18.4 (3C), 12.6 (3C), 10.1; IR (film) 2943, 2860, $1731,1684,1613,1502,1455,1378,1343,1102,1026,908,879 \mathrm{~cm}^{-1}$; HRMS (MALDI-FTMS) calcd for $\left(\mathrm{C}_{39} \mathrm{H}_{54} \mathrm{~N}_{2} \mathrm{O}_{7} \mathrm{Si}\right)[\mathrm{M}+\mathrm{H}]^{+}$691.3773; found 691.3767.

The corresponding intermediate alcohol could also be isolated and characterized: ${ }^{1} \mathrm{H}$ NMR $\left(600 \mathrm{MHz}, \mathrm{C}_{6} \mathrm{D}_{6}\right) \delta 7.10-7.23(\mathrm{~m}, 4 \mathrm{H}), 7.07(\mathrm{t}, J=6.8 \mathrm{~Hz}, 1 \mathrm{H}), 6.41(\mathrm{~d}, J=7.9 \mathrm{~Hz}, 1 \mathrm{H})$, $6.18(\mathrm{~d}, J=7.9 \mathrm{~Hz}, 1 \mathrm{H}), 5.84(\mathrm{~s}, 1 \mathrm{H}), 4.61(\mathrm{~d}, J=11.8 \mathrm{~Hz}, 1 \mathrm{H}), 4.26(\mathrm{~s}, 1 \mathrm{H}), 4.11(\mathrm{~d}, J=11.9$ $\mathrm{Hz}, 1 \mathrm{H}), 4.03(\mathrm{dd}, J=5.5,12.1 \mathrm{~Hz}, 1 \mathrm{H}), 3.80-3.86(\mathrm{~m}, 1 \mathrm{H}), 3.81(\mathrm{~s}, 1 \mathrm{H}), 3.64-3.70(\mathrm{~m}, 1 \mathrm{H})$, $3.55(\mathrm{~s}, 1 \mathrm{H}), 3.39(\mathrm{~s}, 3 \mathrm{H}), 3.35(\mathrm{~s}, 3 \mathrm{H}), 2.58(\mathrm{~s}, 3 \mathrm{H}), 2.40-2.46(\mathrm{~m}, 1 \mathrm{H}), 2.27(\mathrm{dd}, J=5.5,12.9$ $\mathrm{Hz}, 1 \mathrm{H}), 2.04-2.09(\mathrm{~m}, 1 \mathrm{H}), 1.75-1.80(\mathrm{~m}, 1 \mathrm{H}), 1.17-1.23(\mathrm{~m}, 1 \mathrm{H}), 0.68-0.73(\mathrm{~m}, 1 \mathrm{H}), 0.47(\mathrm{t}$, $J=7.2 \mathrm{~Hz}, 3 \mathrm{H}) ;{ }^{13} \mathrm{C}$ NMR $\left(150 \mathrm{MHz}, \mathrm{CDCl}_{3}\right) \delta 172.3,171.3,161.8,153.9,137.7,128.2,127.5$, $126.9,123.6,120.1,107.5,100.9,92.3,87.5,86.1,81.8,74.4,66.0,63.6,55.3,52.9,46.6$, $45.8,38.5,36.3,33.0,19.5,9.7 ;{ }^{13} \mathrm{C} N M R\left(150 \mathrm{MHz}, \mathrm{C}_{6} \mathrm{D}_{6}\right) \delta 172.6,171.5,162.3,154.3,138.5$, 128.4 (2C), 127.6 (2C), 127.0, 124.1, 120.7, 108.0, 101.5, 92.6, 87.8, 86.1, 82.3, 74.2, 66.3, 63.8, 54.7, 52.0, 46.9, 45.9, 38.9, 37.2, 32.9, 19.4, 9.7; IR (film) 3404 (br), 2956, 1728, 1672, $1621,1454,1377,1285,1125 \mathrm{~cm}^{-1}$; HRMS (MALDI-FTMS) calcd for $\left(\mathrm{C}_{30} \mathrm{H}_{34} \mathrm{~N}_{2} \mathrm{O}_{7}\right)[\mathrm{M}+\mathrm{H}]^{+}$ 535.2439; found 535.2438. The relative stereochemistry was confirmed upon X-ray (CCDC 253428) analysis of a crystal grown from EtOAc-hexanes.

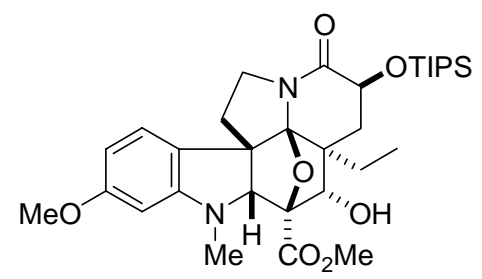

A solution of 18 (304 mg, $0.044 \mathrm{mmol})$ in $\mathrm{MeOH}(22 \mathrm{~mL})$ was stirred under $\mathrm{H}_{2}(1 \mathrm{~atm})$ in the presence of $10 \% \mathrm{Pd} / \mathrm{C}(152 \mathrm{mg})$ for $17 \mathrm{~h}$ at $25{ }^{\circ} \mathrm{C}$. The reaction mixture was filtered through Celite and concentrated in vacuo. Flash chromatography $\left(\mathrm{SiO}_{2}, 25 \%\right.$ EtOAc-hexanes) afforded 19 as a white solid (238 mg, 90\%): ${ }^{1} \mathrm{H}$ NMR $\left(600 \mathrm{MHz}, \mathrm{C}_{6} \mathrm{D}_{6}\right) \delta 6.40(\mathrm{~d}, J=8.3 \mathrm{~Hz}$, 
$1 \mathrm{H}), 6.29(\mathrm{~d}, J=10.5 \mathrm{~Hz}, 1 \mathrm{H}), 6.25(\mathrm{dd}, J=2.2,8.3 \mathrm{~Hz}, 1 \mathrm{H}), 5.95(\mathrm{~d}, J=2.2 \mathrm{~Hz}, 1 \mathrm{H}), 4.54$ (dd, $J=5.3,11.9 \mathrm{~Hz}, 1 \mathrm{H}), 4.41(\mathrm{dd}, J=1.3,10.5 \mathrm{~Hz}, 1 \mathrm{H}), 4.06(\mathrm{~d}, J=1.3 \mathrm{~Hz}, 1 \mathrm{H}), 3.74(\mathrm{dd}, J$ $=4.4,10.1 \mathrm{~Hz}, 2 \mathrm{H}), 3.35(\mathrm{~s}, 3 \mathrm{H}), 3.27(\mathrm{~s}, 3 \mathrm{H}), 2.64-2.69(\mathrm{~m}, 1 \mathrm{H}), 2.59(\mathrm{~s}, 3 \mathrm{H}), 2.46(\mathrm{dd}, J=$ 5.7, $13.2 \mathrm{~Hz}, 1 \mathrm{H}), 1.99-2.05(\mathrm{~m}, 1 \mathrm{H}), 1.57-1.61(\mathrm{~m}, 1 \mathrm{H}), 1.28-1.35(\mathrm{~m}, 3 \mathrm{H}), 1.26(\mathrm{~d}, J=7.4$ $\mathrm{Hz}, 9 \mathrm{H}), 1.22(\mathrm{~d}, J=7.4 \mathrm{~Hz}, 9 \mathrm{H}), 1.02-1.07(\mathrm{~m}, 1 \mathrm{H}), 0.86-0.96(\mathrm{~m}, 5 \mathrm{H}) ;{ }^{13} \mathrm{C} \mathrm{NMR}(150 \mathrm{MHz}$, $\left.\mathrm{C}_{6} \mathrm{D}_{6}\right) \delta 170.6,170.2,161.8,154.5,124.3,123.6,107.9,107.1,98.1,84.4(2 \mathrm{C}), 82.7,68.3$, $63.9,54.9,52.1,46.1,46.0,39.4,38.4,37.4,20.2,18.4$ (3C), 18.3 (3C), 12.9 (3C), 10.0; IR (film) 3307 (br), 2943, 2907, 2860, 1737, 1690, 1584, 1461, 1443, 1378, 1343, 1273, 1161, $1096,1061,1032,885,797 \mathrm{~cm}^{-1}$; HRMS (MALDI-FTMS) calcd for $\left(\mathrm{C}_{32} \mathrm{H}_{48} \mathrm{~N}_{2} \mathrm{O}_{7} \mathrm{Si}\right)[\mathrm{M}+\mathrm{H}]^{+}$ 623.3132; found 623.3123 .

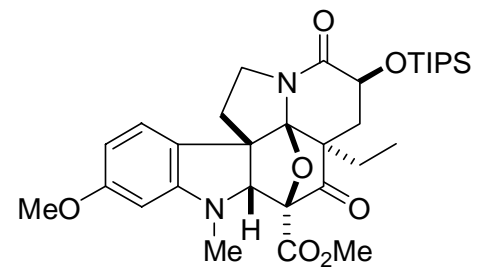

TPAP $(2.8 \mathrm{mg}, 0.008 \mathrm{mmol})$ was added a solution of $19(97 \mathrm{mg}, 0.16 \mathrm{mmol})$, NMO (28 $\mathrm{mg}, 0.24 \mathrm{mmol}$ ), and powered activated $4 \AA$ molecular sieves in anhydrous $\mathrm{CH}_{2} \mathrm{Cl}_{2}(3 \mathrm{~mL})$ at 0 ${ }^{\circ} \mathrm{C}$ under Ar. The mixture was stirred at $0{ }^{\circ} \mathrm{C}$ for $10 \mathrm{~min}$, before warming to $25{ }^{\circ} \mathrm{C}$. Upon disappearance of the starting material (ca. $7 \mathrm{~h}$ ), the mixture was filtered through a short pad of $\mathrm{SiO}_{2}$ eluting with EtOAc. The filtrate was concentrated in vacuo and flash chromatography $\left(\mathrm{SiO}_{2}\right.$, 25\% EtOAc-hexanes) afforded 20 as a white solid (67 mg, 69\%; 60-81\%): ${ }^{1} \mathrm{H}$ NMR (500 MHz, $\left.\mathrm{C}_{6} \mathrm{D}_{6}\right) \delta 6.39(\mathrm{~d}, J=8.0 \mathrm{~Hz}, 1 \mathrm{H}), 6.14(\mathrm{dd}, J=2.0,8.2 \mathrm{~Hz}, 1 \mathrm{H}), 5.87(\mathrm{~d}, J=2.2 \mathrm{~Hz}, 1 \mathrm{H}), 4.54$ (dd, $J=5.8,11.3 \mathrm{~Hz}, 1 \mathrm{H}), 4.14(\mathrm{~s}, 1 \mathrm{H}), 3.87-3.92(\mathrm{~m}, 1 \mathrm{H}), 3.72-3.78(\mathrm{~m}, 1 \mathrm{H}), 3.32(\mathrm{~s}, 3 \mathrm{H})$, $3.29(\mathrm{~s}, 3 \mathrm{H}), 2.96-3.01(\mathrm{~m}, 1 \mathrm{H}), 2.74(\mathrm{~s}, 3 \mathrm{H}), 2.51(\mathrm{dd}, J=5.7,13.4 \mathrm{~Hz}, 1 \mathrm{H}), 2.00-2.07(\mathrm{~m}$, $1 \mathrm{H}), 1.62-1.67(\mathrm{~m}, 1 \mathrm{H}), 1.14-1.27(\mathrm{~m}, 22 \mathrm{H}), 0.98-1.07(\mathrm{~m}, 1 \mathrm{H}), 0.88(\mathrm{t}, J=7.0 \mathrm{~Hz}, 3 \mathrm{H}) ;{ }^{13} \mathrm{C}$ NMR $\left(150 \mathrm{MHz}, \mathrm{C}_{6} \mathrm{D}_{6}\right) \delta 204.3,169.8,166.8,162.5,154.9,123.9,120.4,105.5,103.5,94.4$, 93.9, 80.7, 67.8, 64.2, 54.8, 52.4, 51.7, 47.3, 36.4, 34.2, 34.0, 20.9, 18.3 (3C), 18.2 (3C), 12.9 (3C), 9.2; ${ }^{13} \mathrm{C}$ NMR $\left(150 \mathrm{MHz}, \mathrm{CDCl}_{3}\right) \delta 203.5,170.3,166.2,162.0,154.6,123.6,119.9,104.9$, 103.0, 94.6, 93.4, 80.8, 67.1, 64.0, 55.4, 53.2, 51.3, 47.1, 36.4, 34.4 (2C), 20.2, 18.0 (3C), 17.9 (3C), 12.4 (3C), 9.0; IR (film) 2949, 2855, 1775, 1740, 1693, 1622, 1499, 1458, 1399, 
$1381,1349,1234,1199,1152,1135,1097,1036,1007,883,734,682 \mathrm{~cm}^{-1}$; HRMS (MALDIFTMS) calcd for $\left(\mathrm{C}_{32} \mathrm{H}_{46} \mathrm{~N}_{2} \mathrm{O}_{7} \mathrm{Si}\right)[\mathrm{M}+\mathrm{H}]^{+} 621.2966$; found 621.2945 .

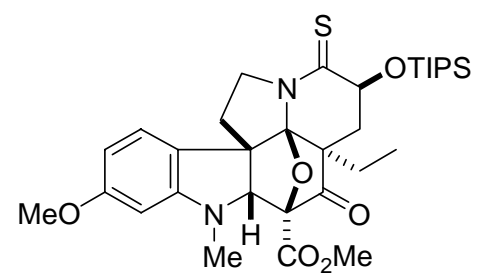

Lawesson's reagent $(28 \mathrm{mg}, 0.71 \mathrm{mmol})$ was added to a toluene solution $(1.8 \mathrm{~mL})$ of 20 (35 mg, $0.059 \mathrm{mmol}$ ) at $25^{\circ} \mathrm{C}$ under Ar. The heterogeneous mixture was warmed to $110^{\circ} \mathrm{C}$ and stirred for $4 \mathrm{~h}$. Upon completion, the now homogeneous yellow solution was cooled to $25{ }^{\circ} \mathrm{C}$ and concentrated onto Celite. Flash chromatography $\left(\mathrm{SiO}_{2}, 20 \%\right.$ EtOAc-hexanes) afforded 21 as a pale yellow solid (25 mg, 69\%, 50-81\%): ${ }^{1} \mathrm{H}$ NMR $\left(600 \mathrm{MHz}, \mathrm{C}_{6} \mathrm{D}_{6}\right) \delta 6.42(\mathrm{~d}, J=8.4 \mathrm{~Hz}$, $1 \mathrm{H}), 6.09(\mathrm{dd}, J=1.4,8.0 \mathrm{~Hz}, 1 \mathrm{H}), 5.87(\mathrm{~d}, J=1.4 \mathrm{~Hz}, 1 \mathrm{H}), 4.74(\mathrm{dd}, J=5.5,10.6 \mathrm{~Hz}, 1 \mathrm{H})$, 4.16-4.21 (m, 2H) , $4.12(\mathrm{~s}, 1 \mathrm{H}), 3.31(\mathrm{~s}, 3 \mathrm{H}), 3.28(\mathrm{~s}, 3 \mathrm{H}), 2.93(\mathrm{t}, J=12.1 \mathrm{~Hz}, 1 \mathrm{H}), 2.72(\mathrm{~s}$, $3 \mathrm{H}), 2.56(\mathrm{dd}, J=5.5,13.2 \mathrm{~Hz}, 1 \mathrm{H}), 2.01-2.09(\mathrm{~m}, 1 \mathrm{H}), 1.66-1.71(\mathrm{~m}, 1 \mathrm{H}), 1.25-1.34(\mathrm{~m}$, $3 \mathrm{H}), 1.20(\mathrm{~d}, J=7.7 \mathrm{~Hz}, 9 \mathrm{H}), 1.15(\mathrm{~d}, J=7.7 \mathrm{~Hz}, 9 \mathrm{H}), 0.86-0.93(\mathrm{~m}, 3 \mathrm{H}) ;{ }^{13} \mathrm{C}$ NMR $(125 \mathrm{MHz}$, $\left.\mathrm{C}_{6} \mathrm{D}_{6}\right) \delta 203.9,203.8,166.5,162.6,155.0,123.9,119.9,103.6,102.5,94.6,94.1,80.7,73.0$, $64.9,54.9,54.8,52.5,52.0,36.0,34.1,34.0,20.9,18.6$ (3C), 18.5 (3C), 13.2 (3C), 9.2; IR (film) 2940, 2865, 1774, 1742, 1621, 1500, 1420, 1360, 1321, 1236, 1205, 1135, 1106, 1006, $881,850 \mathrm{~cm}^{-1}$; HRMS (MALDI-FTMS) calcd for $\left(\mathrm{C}_{32} \mathrm{H}_{46} \mathrm{~N}_{2} \mathrm{O}_{6} \mathrm{SSi}\right)[\mathrm{M}+\mathrm{H}]^{+} 615.2918$; found 615.2920.

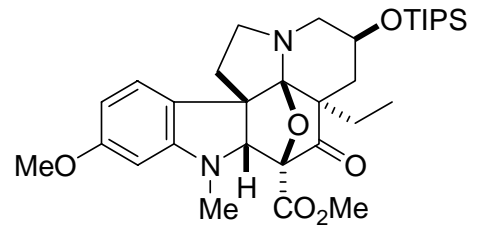

A solution of $21(31 \mathrm{mg}, 0.051 \mathrm{mmol})$ in anhydrous THF $(2.5 \mathrm{~mL})$ was added to excess $\mathrm{Ra}-\mathrm{Ni}$ (pretreated with successive washes in $\mathrm{H}_{2} \mathrm{O}(3 \mathrm{x}), \mathrm{MeOH}(3 \mathrm{x}), \mathrm{THF}(3 \mathrm{x})$ ) under Ar. The mixture was stirred at $25{ }^{\circ} \mathrm{C}$ for $2 \mathrm{~h}$ before being filtered through a pad of Celite, and concentrated under reduced pressure. The residue was taken up in EtOAc and passed through a short plug of $\mathrm{SiO}_{2}$ to provide 22 as a pale yellow oil (18 mg, 61\%; 54-78\%): ${ }^{1} \mathrm{H}$ NMR (600 
$\left.\mathrm{MHz}, \mathrm{C}_{6} \mathrm{D}_{6}\right) \delta 6.83(\mathrm{~d}, J=8.3 \mathrm{~Hz}, 1 \mathrm{H}), 6.27(\mathrm{dd}, J=2.2,8.3 \mathrm{~Hz}, 1 \mathrm{H}), 5.95(\mathrm{~d}, J=2.2 \mathrm{~Hz}, 1 \mathrm{H})$, $4.34(\mathrm{~s}, 1 \mathrm{H}), 4.22-4.27(\mathrm{~m}, 1 \mathrm{H}), 3.34(\mathrm{~s}, 3 \mathrm{H}), 3.30(\mathrm{~s}, 3 \mathrm{H}), 3.21-3.25(\mathrm{~m}, 2 \mathrm{H}), 2.96-3.01(\mathrm{~m}$, $2 \mathrm{H}), 2.87(\mathrm{~s}, 3 \mathrm{H}), 2.39-2.44(\mathrm{~m}, 1 \mathrm{H}), 2.30(\mathrm{dd}, J=4.0,12.7 \mathrm{~Hz}, 1 \mathrm{H}), 2.12-2.18(\mathrm{~m}, 1 \mathrm{H})$, 1.99-2.04 (m, 1H), 0.85-1.20 (m, 26H); ${ }^{13} \mathrm{C}$ NMR $\left(150 \mathrm{MHz}, \mathrm{C}_{6} \mathrm{D}_{6}\right) \delta 207.3,168.2,162.3,154.7$, 124.6, 123.0, 110.9, 103.1, 93.9, 92.6, 82.9, 65.3, 62.5, 54.9, 54.8, 52.2, 51.6, 51.1, 38.8, 35.3, 34.0, 21.9, 18.2 (6C), 12.5 (3C), 10.0; IR (film) 2943, 2865, 1770, 1621, 1500, 1462, 1328, 1252, 1097, 1026, 882, 881, 737, $683 \mathrm{~cm}^{-1}$; HRMS (MALDI-FTMS) calcd for $\left(\mathrm{C}_{32} \mathrm{H}_{48} \mathrm{~N}_{2} \mathrm{O}_{6} \mathrm{Si}\right)[\mathrm{M}+\mathrm{H}]^{+}$585.3354; found 585.3385 .

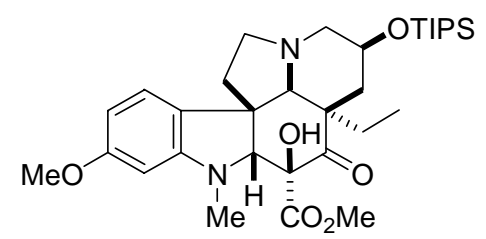

$\mathrm{PtO}_{2}(1-2 \mathrm{mg})$ and a drop of conc. $\mathrm{HCl}$ was added to a solution of $22(8.8 \mathrm{mg}, 0.015$ $\mathrm{mmol})$ in $\mathrm{MeOH}(0.7 \mathrm{~mL})$ at $25{ }^{\circ} \mathrm{C}$. The mixture was stirred under $40-45 \mathrm{psi}$ of $\mathrm{H}_{2}$ for $2 \mathrm{~h}$. The resulting mixture was filtered through a pad of Celite to remove the catalyst, diluted with EtOAc and washed with a saturated aqueous $\mathrm{NaHCO}_{3}$. The aqueous layer was extracted with EtOAC $(2 \mathrm{x})$, the combined organic phase was dried over $\mathrm{Na}_{2} \mathrm{SO}_{4}$, filtered, and concentrated in vacuo. Flash chromatography $\left(\mathrm{SiO}_{2}, 25 \%\right.$ EtOAc-hexanes) afforded 23 as a white solid $(7.2 \mathrm{mg}, 82 \%)$ : ${ }^{1} \mathrm{H}$ NMR $\left(600 \mathrm{MHz}, \mathrm{C}_{6} \mathrm{D}_{6}\right) \delta 7.76(\mathrm{~s}, 1 \mathrm{H}), 6.70(\mathrm{~d}, J=7.9 \mathrm{~Hz}, 1 \mathrm{H}), 6.43(\mathrm{dd}, J=2.0,8.1 \mathrm{~Hz}$, $1 \mathrm{H}), 6.10(\mathrm{~d}, J=1.8 \mathrm{~Hz}, 1 \mathrm{H}), 3.86(\mathrm{~s}, 3 \mathrm{H}), 3.81-3.83(\mathrm{~m}, 1 \mathrm{H}), 3.49(\mathrm{~s}, 3 \mathrm{H}), 3.37(\mathrm{~s}, 3 \mathrm{H}), 2.92$ $(\mathrm{d}, J=11.4 \mathrm{~Hz}, 1 \mathrm{H}), 2.82(\mathrm{t}, J=8.8 \mathrm{~Hz}, 1 \mathrm{H}), 2.68(\mathrm{~d}, J=14.0 \mathrm{~Hz}, 1 \mathrm{H}), 2.45(\mathrm{~s}, 3 \mathrm{H}), 2.29(\mathrm{td}$, $J=10.1,13.6 \mathrm{~Hz}, 1 \mathrm{H}), 2.04-2.08(\mathrm{~m}, 2 \mathrm{H}), 1.93-1.99(\mathrm{~m}, 1 \mathrm{H}), 1.66(\mathrm{~d}, J=11.4 \mathrm{~Hz}, 1 \mathrm{H}), 1.53-$ $1.58(\mathrm{~m}, 1 \mathrm{H}), 1.29-1.35(\mathrm{~m}, 1 \mathrm{H}), 1.08-1.20(\mathrm{~m}, 21 \mathrm{H}), 0.87(\mathrm{dd}, J=3.3,13.8 \mathrm{~Hz}, 1 \mathrm{H}), 0.49(\mathrm{t}$, $J=7.2 \mathrm{~Hz}, 3 \mathrm{H}) ;{ }^{13} \mathrm{C}$ NMR $\left(125 \mathrm{MHz}, \mathrm{C}_{6} \mathrm{D}_{6}\right) \delta 200.8,170.1,161.6,155.5,128.3,123.1,105.7$, $96.8,86.2,80.2,77.1,65.9,59.6,54.9,54.2,51.6(2 \mathrm{C}), 47.3,45.3,40.3,36.9,28.2,18.4(6 \mathrm{C})$, 12.5 (3C), 7.5; IR (film) 2944, 2861, 1749, 1718, 1610, 1595, 1497, 1456, 1246, 1226, 1164, $1092,1072,1020,882,728,677 \mathrm{~cm}^{-1}$; HRMS (MALDI-FTMS) calcd for $\left(\mathrm{C}_{32} \mathrm{H}_{50} \mathrm{~N}_{2} \mathrm{O}_{6} \mathrm{Si}\right)[\mathrm{M}+\mathrm{H}]^{+}$ 587.3511; found 587.3521. 


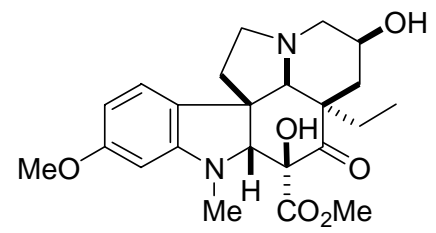

$\mathrm{Bu}_{4} \mathrm{NF}(15 \mu \mathrm{L}, 0.015 \mathrm{mmol}, 1.0 \mathrm{M}$ in THF) was added dropwise to a THF ( $1 \mathrm{~mL})$ solution of $23(5.8 \mathrm{mg}, 0.010 \mathrm{mmol})$ at $0{ }^{\circ} \mathrm{C}$ under Ar. The reaction mixture was stirred for $3 \mathrm{~h}$ at $0{ }^{\circ} \mathrm{C}$ and then slowly warmed to $25{ }^{\circ} \mathrm{C}$ until the starting material was consumed. The mixture was diluted with EtOAC and washed with a saturated aqueous $\mathrm{NH}_{4} \mathrm{Cl}$. The aqueous layer was extracted with EtOAc (2x), and the combined organic layers were dried with $\mathrm{Na}_{2} \mathrm{SO}_{4}$, filtered, and concentrated in vacuo. PTLC $\left(\mathrm{SiO}_{2}, 5 \% \mathrm{MeOH}-\mathrm{CH}_{2} \mathrm{Cl}_{2}\right)$ afforded 24 as a white amorphous solid (4.1 mg, 98\%): ${ }^{1} \mathrm{H}$ NMR $\left(600 \mathrm{MHz}, \mathrm{C}_{6} \mathrm{D}_{6}\right) \delta 8.46(\mathrm{br} \mathrm{s}, 1 \mathrm{H}), 6.98(\mathrm{~d}, J=8.3 \mathrm{~Hz}, 1 \mathrm{H}), 6.37$ (dd, $J=1.6,8.1 \mathrm{~Hz}, 1 \mathrm{H}), 6.13(\mathrm{~d}, J=1.7 \mathrm{~Hz}, 1 \mathrm{H}), 4.05-4.08(\mathrm{~m}, 1 \mathrm{H}), 3.88(\mathrm{~s}, 3 \mathrm{H}), 3.86(\mathrm{~s}$, $3 \mathrm{H}), 3.78(\mathrm{~s}, 3 \mathrm{H}), 3.30-3.35(\mathrm{~m}, 1 \mathrm{H}), 3.26(\mathrm{~d}, J=11.8 \mathrm{~Hz}, 1 \mathrm{H}), 2.67(\mathrm{~s}, 3 \mathrm{H}), 2.64(\mathrm{~d}, J=14.9$ $\mathrm{Hz}, 1 \mathrm{H}), 2.43-2.52(\mathrm{~m}, 3 \mathrm{H}), 2.40(\mathrm{~s}, 1 \mathrm{H}), 2.17(\mathrm{~d}, J=11.8 \mathrm{~Hz}, 1 \mathrm{H}), 1.74($ br s, $1 \mathrm{H}), 1.36-1.44$ $(\mathrm{m}, 1 \mathrm{H}), 1.36(\mathrm{dd}, J=3.3,14.7 \mathrm{~Hz}, 1 \mathrm{H}), 1.21-1.29(\mathrm{~m}, 1 \mathrm{H}), 0.50(\mathrm{t}, J=7.2 \mathrm{~Hz}, 3 \mathrm{H}) ;{ }^{13} \mathrm{C} \mathrm{NMR}$ $\left(125 \mathrm{MHz}, \mathrm{C}_{6} \mathrm{D}_{6}\right) \delta 205.9,169.9,161.1,154.6,127.0,123.0,105.3,96.8,85.1,79.5,76.6,65.2$, 58.0, 55.4, 53.7, 52.6, 51.5, 48.2, 44.6, 40.1, 36.3, 26.8, 7.2; IR (film) 3367 (br), 2934, 2864, $1743,1712,1617,1499,1456,1432,1255,1224,1165,1082,1070,1039 \mathrm{~cm}^{-1}$; HRMS (MALDI-FTMS) calcd for $\left(\mathrm{C}_{23} \mathrm{H}_{30} \mathrm{~N}_{2} \mathrm{O}_{6}\right)[\mathrm{M}+\mathrm{H}]^{+} 431.2177$; found 431.2181 .

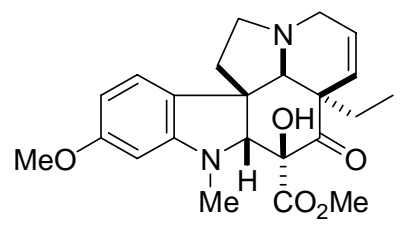

A solution of $24(3.0 \mathrm{mg}, 0.007 \mathrm{mmol})$ and $\mathrm{Ph}_{3} \mathrm{P}(3.7 \mathrm{mg}, 0.014 \mathrm{mmol})$ in anhydrous $\mathrm{CH}_{3} \mathrm{CN}(60 \mu \mathrm{L})$ was warmed to $70{ }^{\circ} \mathrm{C}$. After $10-15 \mathrm{~min}$ at this temperature, anhydrous $\mathrm{CCl}_{4}(40$ $\mu \mathrm{L}$ ) was added dropwise via syringe and the mixture was stirred for an additional $1 \mathrm{~h}$ at $70{ }^{\circ} \mathrm{C}$, before cooling to $25{ }^{\circ} \mathrm{C}$. The solution is diluted with $\mathrm{CH}_{2} \mathrm{Cl}_{2}$ and washed with dilute aqueous $\mathrm{NH}_{4} \mathrm{OH}$. The layers were separated and the aqueous layer was extracted with $\mathrm{CH}_{2} \mathrm{Cl}_{2}(3 \mathrm{x})$. The combined organic layer was dried over $\mathrm{Na}_{2} \mathrm{SO}_{4}$ and concentrated in vacuo. PTLC $\left(\mathrm{SiO}_{2}, 5 \%\right.$ $\mathrm{MeOH}-\mathrm{CH}_{2} \mathrm{Cl}_{2}$ ) afforded 25 as a white amorphous solid $(1.75 \mathrm{mg}, 62 \%)$ identical in all respects 
to material previously reported (J. Org. Chem. 1985, 50, 961): ${ }^{1} \mathrm{H} N M R\left(500 \mathrm{MHz}, \mathrm{CDCl}_{3}\right) \delta$ $6.99(\mathrm{~d}, J=8.4 \mathrm{~Hz}, 1 \mathrm{H}), 6.35(\mathrm{dd}, J=2.2,8.1 \mathrm{~Hz}, 1 \mathrm{H}), 6.11(\mathrm{~d}, J=1.8 \mathrm{~Hz}, 1 \mathrm{H}), 5.79(\mathrm{dd}, J=$ 4.4, $9.9 \mathrm{~Hz}, 1 \mathrm{H}), 5.69(\mathrm{~d}, J=9.9 \mathrm{~Hz}, 1 \mathrm{H}), 3.93(\mathrm{~s}, 1 \mathrm{H}), 3.88(\mathrm{~s}, 3 \mathrm{H}), 3.78(\mathrm{~s}, 3 \mathrm{H}), 3.44-3.58$ $(\mathrm{m}, 2 \mathrm{H}), 2.87(\mathrm{~d}, J=16.1 \mathrm{~Hz}, 1 \mathrm{H}), 2.71(\mathrm{~s}, 1 \mathrm{H}), 2.69(\mathrm{~s}, 3 \mathrm{H}), 2.49-2.59(\mathrm{~m}, 3 \mathrm{H}), 1.49-1.57$ $(\mathrm{m}, 1 \mathrm{H}), 1.28-1.33(\mathrm{~m}, 1 \mathrm{H}), 0.45(\mathrm{t}, J=7.3 \mathrm{~Hz}, 3 \mathrm{H})$; HRMS (MALDI-FTMS) calcd for $\left(\mathrm{C}_{23} \mathrm{H}_{28} \mathrm{~N}_{2} \mathrm{O}_{5}\right)[\mathrm{M}+\mathrm{H}]^{+}$413.2085; found 413.2085.

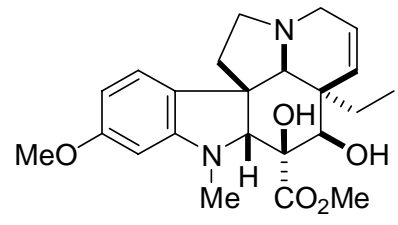

A solution of $25(1.4 \mathrm{mg}, 0.0034 \mathrm{mmol})$ in anhydrous THF ( $38 \mu \mathrm{L})$ at -30 to $-25{ }^{\circ} \mathrm{C}$ was treated with $\mathrm{AlCl}_{3}(5.4 \mathrm{mg}, 0.04 \mathrm{mmol})$. The mixture was stirred and sodium bis(2methoxyethoxy)aluminium hydride ( $86 \mu \mathrm{L}$ of a $0.0512 \mathrm{M}$ solution in THF) was added dropwise. The temperature was slowly raised to $-20^{\circ} \mathrm{C}$. Upon completion of the reaction based on TLC, the solution was diluted with EtOAc, and washed with $\mathrm{H}_{2} \mathrm{O}$. The aqueous layer was extracted with EtOAc (3x), dried over $\mathrm{Na}_{2} \mathrm{SO}_{4}$, filtered, and concentrated in vacuo. PTLC ( $\mathrm{SiO}_{2}, 5 \%$ $\mathrm{MeOH}-\mathrm{CH}_{2} \mathrm{Cl}_{2}$ ) afforded 26 as a film $(0.6 \mathrm{mg}, 43 \% ; 43-50 \%)$ identical in all respects to material previously reported (Collect. Czech. Chem. Commun. 2000, 65, 789) and identical to authentic material prepared from naturally occurring vindoline: ${ }^{1} \mathrm{H} \mathrm{NMR}\left(600 \mathrm{MHz}, \mathrm{CDCl}_{3}\right) \delta 9.24$ $(\mathrm{s}, 1 \mathrm{H}), 6.87(\mathrm{~d}, J=7.9 \mathrm{~Hz}, 1 \mathrm{H}), 6.28(\mathrm{dd}, J=2.2,7.9 \mathrm{~Hz}, 1 \mathrm{H}), 6.06(\mathrm{~d}, J=2.2 \mathrm{~Hz}, 1 \mathrm{H}), 5.87$ $(\mathrm{dd}, J=3.5,10.5 \mathrm{~Hz}, 1 \mathrm{H}), 5.74(\mathrm{~d}, J=10.1 \mathrm{~Hz}, 1 \mathrm{H}), 4.08(\mathrm{~d}, J=7.0 \mathrm{~Hz}, 1 \mathrm{H}), 3.84(\mathrm{~s}, 3 \mathrm{H})$, $3.78(\mathrm{~s}, 3 \mathrm{H}), 3.72(\mathrm{~s}, 1 \mathrm{H}), 3.36-3.50(\mathrm{~m}, 2 \mathrm{H}), 2.83-2.89(\mathrm{~m}, 1 \mathrm{H}), 2.72(\mathrm{~s}, 3 \mathrm{H}), 2.66(\mathrm{~s}, 1 \mathrm{H})$, 2.49-2.62 (m, 2H), 2.19-2.28 (m, 2H), 1.44-1.51 (m, 1H), 0.98-1.04 (m, 1H), $0.67(\mathrm{t}, J=7.4$ $\mathrm{Hz}, 3 \mathrm{H}) ; \mathrm{HRMS}$ (MALDI-FTMS) calcd for $\left(\mathrm{C}_{23} \mathrm{H}_{30} \mathrm{~N}_{2} \mathrm{O}_{5}\right)[\mathrm{M}+\mathrm{H}]^{+} 415.2227$; found 415.2222.

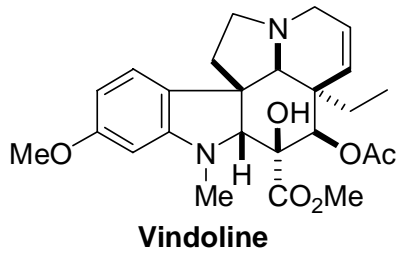


A solution of deacetylvindoline $(\mathbf{2 6}, 1.2 \mathrm{mg}, 0.0029 \mathrm{mmol})$ in $\mathrm{Ac}_{2} \mathrm{O}(80 \mu \mathrm{L})$ at $25^{\circ} \mathrm{C}$ was treated with NaOAC $(1.2 \mathrm{mg}, 0.014 \mathrm{mmol})$. The reaction mixture was stirred at $25{ }^{\circ} \mathrm{C}$ for $2 \mathrm{~h}$, before being diluted with $\mathrm{CH}_{2} \mathrm{Cl}_{2}$. The $\mathrm{pH}$ was adjusted to 10 with addition of saturated aqueous $\mathrm{NaHCO}_{3}$, the layers were separated and the aqueous layer was extracted with $\mathrm{CH}_{2} \mathrm{Cl}_{2}$ (3x). The combined organic layers were dried over $\mathrm{MgSO}_{4}$ and the solvent was removed under reduced pressure. PTLC $\left(\mathrm{SiO}_{2}\right.$, EtOAC) afforded vindoline (3) as a white amorphous solid (1.1 mg, 83\%) identical in all respects with authentic material: ${ }^{1} \mathrm{H}$ NMR $\left(600 \mathrm{MHz}, \mathrm{CDCl}_{3}\right) \delta 9.55(\mathrm{~s}, 1 \mathrm{H}), 6.89$ $(\mathrm{d}, J=7.9 \mathrm{~Hz}, 1 \mathrm{H}), 6.29(\mathrm{dd}, J=1.8,7.9 \mathrm{~Hz}, 1 \mathrm{H}), 6.08(\mathrm{~d}, J=2.2 \mathrm{~Hz}, 1 \mathrm{H}), 5.85(\mathrm{dd}, J=4.6$, $10.3 \mathrm{~Hz}, 1 \mathrm{H}), 5.46(\mathrm{~s}, 1 \mathrm{H}), 5.24(\mathrm{~d}, J=8.8 \mathrm{~Hz}, 1 \mathrm{H}), 3.79(\mathrm{~s}, 3 \mathrm{H}), 3.78(\mathrm{~s}, 3 \mathrm{H}), 3.75(\mathrm{~s}, 3 \mathrm{H})$, 3.37-3.55 (m, 2H) , 2.77-2.87 (m, $1 \mathrm{H}), 2.67(\mathrm{~s}, 3 \mathrm{H}), 2.46-2.59(\mathrm{~m}, 1 \mathrm{H}), 2.25-2.41(\mathrm{~m}, 2 \mathrm{H})$, $2.07(\mathrm{~s}, 3 \mathrm{H}), 2.04(\mathrm{~s}, 1 \mathrm{H}), 1.60-1.71(\mathrm{~m}, 1 \mathrm{H}), 1.06-1.19(\mathrm{~m}, 1 \mathrm{H}), 0.48(\mathrm{t}, J=7.2 \mathrm{~Hz}, 3 \mathrm{H})$; HRMS (MALDI-FTMS) calcd for $\left(\mathrm{C}_{25} \mathrm{H}_{32} \mathrm{~N}_{2} \mathrm{O}_{6}\right)[\mathrm{M}+\mathrm{H}]^{+}$457.2333; found 457.2313.

\section{References}

(S1) Woodward, R. B.; Bader, F. E.; Bickel, H.; Frey, A. J.; Kierstead, R. W. Tetrahedron 1958, $2,1$.

(S2) Feldman, P. L. Rapoport, H. Synthesis 1986, 735.

(S3) He, F.; Bo, Y.; Altom, J. D. Corey, E. J. J. Am. Chem. Soc. 1999, 121, 6771.

(S4) Other synthetic routes to methyl 4-ketohexanoate: (a) Mori, K.; Takikawa, H.; Nishimura, Y. ; Horikiri, H. Liebigs. Ann. Chem. 1997, 327. (b) Banwell, M. G.; Edward, A. J.; Jolliffe, K. A.; Smith, J. A.; Hamel, E.; Verdier-Pinard, P. Org. Biomol. Chem. 2003, 1, 296.

(S5) (a) Moza, B., K.; Trojanek, J. Chem. Industry 1962, 4, 1425-1427. (b) Norbert N. Bull. Chim. Soc. France 1963, 1509 